\title{
Signaling from maize organ primordia via FASCIATED EAR3 regulates stem cell proliferation and yield traits
}

Byoung Il Je ${ }^{1}$, Jeremy Gruel ${ }^{2,7}$, Young Koung Lee ${ }^{1,7}$, Peter Bommert ${ }^{1}$, Edgar Demesa Arevalo ${ }^{1}$, Andrea L. Eveland ${ }^{1}$, Qingyu $\mathbf{W u}^{1}$, Alexander Goldshmidt ${ }^{1}$, Robert Meeley ${ }^{3}$, Madelaine Bartlett $^{4}$, Mai Komatsu ${ }^{5}$, Hajime Sakai ${ }^{5}$, Henrik Jönsson ${ }^{2,6}$ and David Jackson ${ }^{1}$

${ }^{1}$ Cold Spring Harbor Laboratory, Cold Spring Harbor, NY, USA.

${ }^{2}$ Sainsbury Laboratory, Cambridge University, Cambridge, UK.

${ }^{3}$ DuPont Pioneer, Agricultural Biotechnology, Johnston, IA, USA.

${ }^{4}$ Biology Department, University of Massachusetts, Amherst, MA, USA.

${ }^{5}$ DuPont Pioneer, Agricultural Biotechnology, Wilmington DE, USA.

${ }^{6}$ Computational Biology \& Biological Physics Group, Lund University, Lund, Sweden.

${ }^{7}$ These authors contributed equally to this work.

Correspondence should be addressed to D. J. (jacksond@cshl.edu). 
Shoot apical meristems are stem cell niches that balance proliferation with the incorporation of daughter cells into organ primordia. This balance is maintained by CLAVATA-WUSCHEL feedback signaling between the stem cells at the tip of the meristem, and the underlying organizing center. Signals that provide feedback from organ primordia to control the stem cell niche in plants have also been hypothesized, but their identity is unknown. Here we report FASCIATED EAR3 (FEA3), a leucine-rich repeat receptor that functions in stem cell control, and responds to a CLAVATA3/ESR-related (CLE) peptide expressed in organ primordia. We modeled our results to reveal a regulatory system that transmits signals from differentiating cells in organ primordia back to the stem cell niche, and that appears to function broadly in the plant kingdom. Furthermore, we demonstrate an application of this new signaling feedback, by showing that weak alleles of $f e a 3$ enhance hybrid maize yield traits. 
Stem cells occur in niches, specialized microdomains where proliferation of pluripotent cells is controlled by signals from neighboring cells ${ }^{1,2}$. In plants, for example, the WUSCHEL (WUS) transcription factor promotes stem cell fate, and is expressed in the organizing center cells below the stem cells. WUS activates CLAVATA (CLV) gene expression, and the CLV3 peptide signal is secreted from stem cells at the tip of the SAM, is perceived by leucine-rich repeat (LRR) receptors, such as CLV1, expressed in and above the WUS domain, and signals in a feedback to repress WUS $\operatorname{expression}^{3-5}$.

Although such feedback systems are well described in animals and plants, it is not known if or how signals expressed in differentiating progeny cells also feedback to control the stem cell niche. In plants, such signals have been hypothesized ${ }^{6-8}$, and consistent with this idea the inhibition of primordium growth leads to SAM enlargement ${ }^{9}$, but the underlying molecular mechanism(s) are unknown. To search for factors controlling stem cell proliferation, we focused on maize fasciated ear (fea) mutants, some of which correspond to CLV receptors already described in dicots ${ }^{10-13}$. Here we provide evidence to support a new receptor-ligand signaling pathway that acts to restrict stem cell proliferation using a signal expressed in differentiating cells in organ primordia.

\section{RESULTS}

\section{fea3 mutants have enlarged and fasciated meristems.}

The fea 3 mutant arose in an irradiation mutagenesis screen, and was introgressed into the B73 background. fea 3 vegetative shoot apical meristems (SAMs) were wider (mean $150 \pm 4.5 \mu \mathrm{m}$, compared to $128 \pm 5.8 \mu \mathrm{m}$ for wild type (wt) siblings, $P$ value $<0.001$ by 2 tailed $t$ test) (Fig. 1a, b). Despite the larger SAM, its identity and organization was not adversely affected, as shown by KNOTTED1 expression (Supplementary Fig. 1a) ${ }^{14}$. fea 3 mutants had prominent inflorescence defects, including thick tassels, and strongly fasciated ears with increased seed row number, resembling maize $c l v$ mutants $(\text { Fig. } \mathbf{1 c}-\mathbf{j})^{10,13}$, although overall plant stature was normal (Supplementary Fig. 1b). In contrast to maize $c l v$ receptor mutants, however, spikelet and flower development was normal in $f e a 3$ (Supplementary Fig. 1c, d) ${ }^{10,13}$. Fasciated ears and thick tassels 
result from increased stem cell proliferation in inflorescence meristems. We found that fea 3 tassel inflorescence meristems were larger than wt (Fig. 1c, d). fea 3 ear inflorescence meristems were also enlarged, and became more severely affected during development (Fig. 1f-i).

\section{FEA3, a transmembrane receptor, is expressed in the SAM.}

We mapped fea 3 to a region containing $\sim 20$ genes (Fig. 1k), including one that encoded an LRR receptor-like protein. Based on similar phenotypes for related genes ${ }^{10,13}$, we sequenced this locus from fea3-0, and found a retrotransposon fragment inserted in the second exon, introducing a premature stop codon (Supplementary Fig. 2a). Three additional fea3 alleles from a targeted ethylmethane sulphonate (EMS) screen, contained amino acid substitutions relative to the progenitor (Fig. 11 and Supplementary Fig. 2b). Therefore FEA3 encodes an LRR receptor-like protein, with a predicted signal peptide followed by 12 LRRs, a transmembrane domain, and a short cytoplasmic tail lacking any kinase or other predicted signaling domain (Fig. 11).

FEA3 expression was enriched in meristems (Supplementary Fig. 2c), and in situ hybridization detected expression in the inner cells of the vegetative SAM central zone and cells below the central zone, as well as in leaf primordia (Fig. 2a and Supplementary Fig. 2d). FEA3 was also expressed in the central region of ear spikelet meristems (Supplementary Fig. 2f). We next expressed FEA3-red fluorescent protein (RFP) fusions driven by the endogenous promoter. RFP was fused after the signal peptide in one construct, or at the C terminus in another (Supplementary Fig. 3a), and both transgenes rescued fea 3 phenotypes (Supplementary Fig. 3b). FEA3-RFP accumulated in the central region of the SAM (Supplementary Fig. 3c), consistent with the in situ data, but was not enriched in the plasma membrane, which might reflect endocytic trafficking, as reported for CLV ${ }^{15}$. We therefore treated with D15 peptide, an endocytosis inhibitor ${ }^{16}$, and FEA3-RFP became enriched at the cell periphery after $2 \mathrm{hr}$ (Fig. 2b and Supplementary Fig. 3d), and we confirmed its plasma membrane localization biochemically (Supplementary Fig. 3e). FEA3-RFP was detected in the inner layers of the inflorescence SAM (Fig. 2b) in a pattern similar to that observed for FEA3 mRNA in the vegetative SAM (Fig. 2a), starting from the 5-6th layer below the L1 to the 12-13th 
layer of cells. Expression was also found in tassel and ear inflorescence meristems, in spikelet meristems (Supplementary Fig. 3f-h), in leaf primordia (inset, Fig. 2b) and in cells above the quiescent center of the root (Supplementary Fig. 3i).

Since FEA3 was expressed lower down in the SAM compared to known Arabidopsis CLV signaling components ${ }^{12,17}$, we investigated its effect on WUS expression. CLV3 and CLV1 are expressed above and overlapping with the WUS domain, and WUS expression expands upwards in $c l v$ mutants ${ }^{3,4}$. We generated a maize WUS reporter using the ZmWUS1 promoter and 3' region ${ }^{18}$ to drive

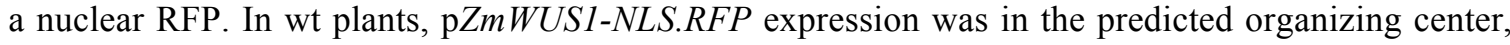
as expected, starting 4-5 cell layers below the L1, and extending down $\sim 5$ cell layers (Fig. $2 \mathbf{c}$ ), in a domain that largely overlapped with FEA3-RFP, though FEA3-RFP expression also extended lower down, below the organizing center. Strikingly, $\mathrm{pZmWUS1-NLS.RFP}$ expression expanded downwards in fea 3 , below the presumptive organizing center (Fig. 2d). Interestingly, despite this obvious downward spread of pZmWUS1 reporter expression, there was no expansion into the upper layers of the SAM (Fig. 2d), suggesting that FEA3 functions to suppress ZmWUS1 expression specifically in the region below the organizing center.

\section{fea3 showed resistance to a novel CLE peptide, ZmFCP1.}

As FEA3 encoded an LRR receptor-like protein, we reasoned that it might perceive CLV3 or a CLV3 related (CLE) peptide. We therefore treated seedlings with peptides, and measured root growth inhibition ${ }^{19,20}$. Maize roots treated with a control, scrambled CLV3 peptide (sCLV3) grew normally, but were inhibited in the presence of $\mathrm{CLV}^{21}$. However, fea2 (maize $\operatorname{clv} 2$ ) mutant roots showed resistance to CLV3 peptide, suggesting that FEA2 functions in CLV3 perception. fea 3 mutants, however, did not show resistance to CLV3 (Supplementary Fig. 4), suggesting FEA3 might perceive a different ligand. We constructed a phylogeny of maize CLV3/ESR-related (ZmCLE) peptides $^{22}$ (Supplementary Fig. 5), and the closest to CLV3 were ZmCLE7 and 14, and rice FLORAL ORGAN NUMBER2 (FON2) ${ }^{23}$. Sister to this clade, we identified a gene orthologous to rice FON2-LIKE CLE PROTEIN1 (FCP1) ${ }^{24}$, that we named ZmFCP1. Rice $f c p 1$ mis-expression lines have 
meristem size phenotypes, but genetic evidence suggests that the rice CLV1 ortholog is not the FCP1 receptor ${ }^{24}$. The predicted peptides encoded by these genes, and additional related peptides were tested, and each of them inhibited wt root growth, similar to CLV3. Interestingly, fea3 roots showed resistance only to ZmFCP1 peptide (Supplementary Fig. 6). We next asked if FEA3 showed similar specificity for ZmFCP1 peptide in the shoot, by measuring its effect on SAM size in cultured embryos $^{21}$. Wt embryos grew normally in the presence of the scrambled CLV3 peptide control, but showed significant decrease of SAM size in presence of ZmFCP1 or related peptides, such as ZmCLE7 or ZmCLE14, whereas fea3 again showed resistance only to ZmFCP1 (Fig. 3a, b). These findings suggest that FEA3 functions as a receptor in perception of the $\mathrm{ZmFCP1}$ peptide. In contrast, fea 2 mutants showed resistance to a broader class of peptides, including CLV3, ZmFCP1 and ESR2c, consistent with findings for clv2 in Arabidopsis (Supplementary Fig. 6b).

In Arabidopsis, CLV3 is expressed in stem cells at the tip of the SAM, and it is believed that CLV3 peptide moves down towards the organizing center to restrict WUS expression ${ }^{3-5}$, although the peptide itself has never been localized in vivo. In contrast to $C L V 3$, we found that $Z m F C P 1$ was not expressed in the SAM, but in leaf primordia and in cells on the flank of the SAM that are destined to be incorporated into primordia (Fig. 3c, for comparison of $F E A 3$ and $Z m F C P 1$ expression see Supplementary Fig. 7a, b). We next obtained a Mutator insertion ${ }^{25}$ in the first exon of $Z m F C P 1$, and homozygous mutants had a fasciated ear phenotype (Fig. 3d). Zmfcpl vegetative SAMs were also wider than normal (mean $167 \pm 10.4 \mu \mathrm{m}$, compared to $142 \pm 7.5 \mu \mathrm{m}$ for wt siblings; $P$ value $<0.001$ ), and $f e a 3$ was epistatic to Zmfcpl, as the double mutants had the same SAM size as fea 3 single mutants (Fig. 3e, f).

In summary, our data support the idea that $Z m F C P 1$ controls SAM size in the same pathway as FEA3. We propose a model in which $\mathrm{ZmFCP1}$ peptide is produced from differentiating primordium cells, and suppresses WUS expression and stem cell proliferation via the FEA3 receptor in the SAM. This model is supported by FEA3 and ZmFCP1 expression patterns, and by our observation of downward spread of WUS in fea 3 mutant meristems. 


\section{Computational modeling of $F E A 3-Z m F C P 1$ interactions.}

To understand the spatial patterning resulting from this new CLE-peptide/ receptor signaling, we integrated the FEA3/ ZmFCP1 regulation of ZmWUS1 with the canonical CLV3/WUS feedback loop, recently modeled in Arabidopsis ${ }^{26-28}$ (methods, Supplementary Fig. 7-12). We assumed that certain functions and expression domains, such as for cytokinin genes, WUS and its activation of stem cells, are conserved in maize, though some of these have not been demonstrated yet. On a 3D geometry representing the maize SAM and two primordia (Supplementary Fig. 7-12), the model reproduced the wt expression domain of $Z m W U S 1$, and could generate a stem cell domain expressing a hypothetical $\mathrm{ZmCLV3}$ ortholog at the central zone (Fig. 4a). The model predicts that a mutation in the FEA3 receptor that responds to a CLE signal from the leaf primordia, leads to downward spread of ZmWUS1 expression, and that this would lead to an expansion of the stem cell population and upregulation of CLV3 expression, which has previously been correlated to fasciation ${ }^{3,29}$. We tested this model in three ways. First, we measured expression of ZmCLE7, a maize ortholog of CLV3 (Supplementary Fig. 5), and found an increase in expression in meristem tips of fea 3 mutants (Supplementary Fig. 13), consistent with our predictions. Next, we characterized fea3; fea 2 double mutants, and found highly synergistic phenotypes in ear inflorescence meristem size, or tassel spikelet density $(P$ value $<0.001)$, a proxy for tassel inflorescence meristem size ${ }^{21}(\mathbf{F i g} . \mathbf{4 b}, \mathbf{c})$, suggesting FEA3 and FEA2 act in different pathways that may converge on the same downstream target, consistent with our model (Fig. 4a). As a third test of the model, we overexpressed ZmFCP1 in organ primordia using a two-component driver system $^{30}$. We used a $p Y A B B Y 14-L h G 4$ line to drive primordium specific expression of a $p O p-Z m F C P 1$ responder line. Driver specificity was confirmed by detection of $p O p-N L S . R F P$ only in primordia (Supplementary Fig. 14a, b), and we measured SAM size in segregating families. Plants carrying either driver or responder components alone had normal SAM size, but plants carrying both constructs showed strong inhibition of SAM growth (Fig. 4d-g), and ZmFCP1 over-expression was confirmed by qRT-PCR (Supplementary Fig. 14c). These tests of the model support the idea that $\mathrm{ZmFCP} 1$ signaling from differentiating primordia is sufficient 
to suppress stem cell proliferation, consistent with a reduced WUS expression domain in the $f c p 1$ over-expression model (Fig. 4a).

\section{Arabidopsis FEA3 ortholog mutants are also fasciated.}

To ask if FEA3 was specific to maize, we characterized its three potential orthologs, Atlg68780, Atlg13230 and At3g25670 in Arabidopsis (Supplementary Fig. 5). Predicted null TDNA mutants of the first two showed no obvious phenotype, even when combined in a double mutant. We therefore made artificial microRNA (Supplementary Fig. 15a, b) or RNAi lines for the third ortholog, and more than $50 \%$ of the RNAi plants showed fasciated inflorescence stems (Fig. $\mathbf{5 b}$, c). RNAi specificity for At3g25670 (hereafter called AtFEA3), was confirmed by qRT-PCR (Supplementary Fig. 15c). As expected, the SAMs of these RNAi lines were enlarged (strong lines $294 \pm 24 \mu \mathrm{m}$, weak lines $85 \pm 12 \mu \mathrm{m}$, compared to $67 \pm 7.6 \mu \mathrm{m}$ in wt; $P$ value $<0.001)$ (Fig. 5d, e). However, in contrast to known $c l v$ mutants ${ }^{31-33}$, but similar to maize fea3, flower and silique development was normal (Supplementary Fig. 15d-f). This might explain why this mutant has not been characterized previously, since it doesn't show the silique/ flower phenotypes typical of $c l v$ mutants. We also asked if AtFEA3 RNAi plants showed altered sensitivity to CLE peptides ${ }^{19}$. Interestingly, as in maize, the AtFEA3 RNAi lines did not show resistance to CLV3, but were resistant to a related CLE peptide, CLE27, in roots and in the SAM (Fig. 5f, g). CLE27 is not the ZmFCP1 ortholog (Supplementary Fig. 5), however it is expressed similarly, at the SAM periphery and not in the central zone (Supplementary Fig. 15g). Our results indicate that FEA3 function is conserved in Arabidopsis, and that it may perceive a CLE peptide that is produced in cells that are programmed for differentiation.

\section{fea3 weak alleles enhance hybrid maize yield traits.}

We previously found that weak alleles of fasciated ear mutants could improve maize yield traits, such as kernel row number ${ }^{34}$, by increasing meristem size and number of primordia while maintaining structural integrity of the meristem. To ask if fea 3 weak alleles have similar effects, we 
backcrossed EMS alleles developed in our non-complementation screen to W22 or B73 inbred lines, and generated F1 hybrids. As expected, the B73/ W22 wt hybrids had significantly enhanced yields compared to the inbreds, due to heterosis. We found that $f e a 3-2$ and $f e a 3-3$ weak allele hybrids further enhanced ear length, kernel row number, kernel number per ear and ear weight (Fig. 6a-f and Supplementary Table 1). The weak alleles were mildly fasciated, but did not display the stunted ear growth that is normally associated with strongly fasciated mutants. These results are particularly exciting, because in our previous studies of weak fea 2 alleles we found an increase in kernel row number but no overall increase in ear weight, due to a compensatory reduction in kernel size ${ }^{34}$. Therefore the newly identified FEA3 signaling pathway could be used to develop new alleles for crop improvement.

\section{DISCUSSION}

Our results reveal a conserved signaling system that has fundamentally different expression patterns and receptor/ peptide combination compared to the well-characterized $C L V$-WUS system. We hypothesize that this system allows feedback control of SAM size using a CLE signal expressed in differentiating primordia. Our model suggests that this new CLE signal moves from the organ primordia to the SAM, where it is perceived by FEA3; however we cannot rule out the possibility of a secondary mobile signal, since the FEA3 receptor is also expressed in primordia. Indeed such a caveat also applies to CLV3, because technical limitations have precluded the localization of CLV3 peptide in vivo. A feedback signaling system from primordia could provide control of stem cell proliferation, and plant growth, by integrating metabolic or other signals from the developing primordia. Weak alleles of $f e a 3$ displayed enhanced yield traits, as well as overall yield, in contrast to weak alleles of $f e a 2$ which increase kernel number without enhancing overall yield ${ }^{34}$, suggesting that fea 3 weak alleles might also enhance sink strength in developing ears. A recent study also highlighted the importance of feedback signaling from progeny cells to the stem cell niche in mammalian hair follicles ${ }^{35}$, suggesting this may be a universal mechanism to control stem cell proliferation during development, with the potential to enhance crop yield traits. 
URLs. Sequences and molecular markers available at MaizeGDB, http://www.maizegdb.org/; twocomponent driver system, http://maize.jcvi.org/cellgenomics/geneDB list.php; FACS sorting data in the Arabidopsis eFP browser, http://bbc.botany.utoronto.ca/efp/cgi-bin/efpWeb.cgi; Python scientific package for modeling, scipy, http://www.scipy.org/.

\section{METHODS}

Methods and any associated references are available in the online version of the paper.

ACCESSION CODES. $f e a 3-0$ sequence is available from GenBank accession number, KX009408 (http://www.ncbi.nlm.nih.gov/Genbank/).

Note: Any Supplementary Information and Source Data files are available in the Online version of the paper.

\section{ACKNOWLEDGEMENTS}

The fea3-0 allele was kindly provided by Victor Shcherbak, Krasnodar Res. Inst. Agric., Russia. We acknowledge funding from a collaborative agreement with Dupont Pioneer, and from NSF Plant Genome Research Program grant \# IOS-1238202 and MCB-1027445, and with the support of the Gatsby Charitable Foundation (GAT3395/PR4) and Swedish Research Council (VR2013-4632) to HJ, and "Next-Generation BioGreen 21 Program (SSAC, Project No. PJ01137901)" Rural Development Administration, Republic of Korea. We also thank Ulises Hernandez for assistance with cloning, Amandine Masson for assistance with peptide assays, and members of the Jackson lab for comments on the manuscript. 


\section{AUTHOR CONTRIBUTIONS}

B.I.J. performed all experimental procedures, except for those listed below, prepared figures and wrote the draft of the manuscript. Y.K.L and P.B. mapped and cloned FEA3. J.G. performed computational modeling, supervised by H.J. E.D.A. analyzed transactivation lines, using constructs generated by Q.W. A.L.E. performed expression analyses. A.G. performed ZmWUS1 reporter line construction and characterization. R.M. provided the $Z m F C P 1$ mutants. M.B. performed phylogenetic analyses. M.K. and H.S. provided field genetics and mapping support and analysis. D.J. supervised the research and co-wrote the manuscript.

\section{COMPETING FINANCIAL INTERESTS}

The authors (B.I.J., Y.K.L., D.J. M.K. and H.S., on behalf of Cold Spring Harbor Laboratory and DuPont Pioneer) have obtained a patent US20150047071 A1 based in part on this work with the US Patent and Trademark Office.

\section{References}

1. Morrison, S.J. \& Spradling, A.C. Stem cells and niches: mechanisms that promote stem cell maintenance throughout life. Cell 132, 598-611 (2008).

2. Heidstra, R. \& Sabatini, S. Plant and animal stem cells: similar yet different. Nat Rev Mol Cell Biol 15, 301-12 (2014).

3. Brand, U., Fletcher, J.C., Hobe, M., Meyerowitz, E.M. \& Simon, R. Dependence of stem cell fate in Arabidopsis on a feedback loop regulated by CLV3 activity. Science 289, 617-9 (2000).

4. Schoof, H. et al. The stem cell population of Arabidopsis shoot meristems is maintained by a regulatory loop between the CLAVATA and WUSCHEL genes. Cell 100, 635-644 (2000).

5. Stahl, Y. \& Simon, R. Plant primary meristems: shared functions and regulatory mechanisms. Curr Opin Plant Biol 13, 53-8 (2010).

6. Emery, J.F. et al. Radial patterning of Arabidopsis shoots by class IIIHD-ZIP and KANADI genes. Current Biology 13, 1768-1774 (2003).

7. Goldshmidt, A., Alvarez, J.P., Bowman, J.L. \& Eshed, Y. Signals derived from YABBY gene activities in organ primordia regulate growth and partitioning of Arabidopsis shoot apical meristems. Plant Cell 20, 1217-1230 (2008).

8. Tanaka, W. et al. The YABBY gene TONGARI-BOUSHI1 is involved in lateral organ development and maintenance of meristem organization in the rice spikelet. Plant Cell 24, 80-95 (2012).

9. Scanlon, M.J. The polar auxin transport inhibitor N-1-naphthylphthalamic acid disrupts leaf initiation, KNOX protein regulation, and formation of leaf margins in maize. Plant Physiol 133, 597-605 (2003).

10. Taguchi-Shiobara, F., Yuan, Z., Hake, S. \& Jackson, D. The fasciated ear2 gene encodes a leucine-rich repeat receptor-like protein that regulates shoot meristem proliferation in maize. Genes \& Development 15, 2755-2766 (2001).

11. Jeong, S., Trotochaud, A.E. \& Clark, S.E. The Arabidopsis CLAVATA2 gene encodes a receptor-like protein required for the stability of the CLAVATA1 receptor-like kinase. Plant Cell 11, 1925-34 (1999).

12. Clark, S.E., Williams, R.W. \& Meyerowitz, E.M. The CLAVATA1 gene encodes a putative receptor kinase that controls shoot and floral meristem size in Arabidopsis. Cell 89, 575-85 (1997).

13. Bommert, P. et al. thick tassel dwarf1 encodes a putative maize ortholog of the Arabidopsis CLAVATA1 leucine-rich repeat receptor-like kinase. Development 132, 1235-45 (2005).

14. Jackson, D., Veit, B. \& Hake, S. Expression of Maize Knotted1 Related Homeobox Genes in the Shoot Apical Meristem Predicts Patterns of Morphogenesis in the Vegetative Shoot. Development 120, 405413 (1994). 
15. Nimchuk, Z.L., Tarr, P.T., Ohno, C., Qu, X.A. \& Meyerowitz, E.M. Plant Stem Cell Signaling Involves Ligand-Dependent Trafficking of the CLAVATA1 Receptor Kinase. Current Biology 21, 345-352 (2011).

16. Luscher, C. et al. Role of AMPA receptor cycling in synaptic transmission and plasticity. Neuron 24, 649-658 (1999).

17. Fletcher, J.C., Brand, U., Running, M.P., Simon, R. \& Meyerowitz, E.M. Signaling of cell fate decisions by CLAVATA3 in Arabidopsis shoot meristems. Science 283, 1911-4 (1999).

18. Nardmann, J. \& Werr, W. The shoot stem cell niche in angiosperms: Expression patterns of WUS orthologues in rice and maize imply major modifications in the course of mono- and dicot evolution. Molecular Biology and Evolution 23, 2492-2504 (2006).

19. Kondo, T. et al. A plant peptide encoded by CLV3 identified by in situ MALDI-TOF MS analysis. Science 313, 845-848 (2006).

20. Ito, Y. et al. Dodeca-CLE peptides as suppressors of plant stem cell differentiation. Science 313, 842-5 (2006).

21. Bommert, P., Je, B.I., Goldshmidt, A. \& Jackson, D. The maize Galpha gene COMPACT PLANT2 functions in CLAVATA signalling to control shoot meristem size. Nature 502, 555-8 (2013).

22. OpsahlFerstad, H.G., LeDeunff, E., Dumas, C. \& Rogowsky, P.M. ZmEsr, a novel endosperm-specific gene expressed in a restricted region around the maize embryo. Plant Journal 12, 235-246 (1997).

23. Suzaki, T. et al. Conservation and diversification of meristem maintenance mechanism in Oryza sativa: Function of the FLORAL ORGAN NUMBER2 gene. Plant Cell Physiol 47, 1591-602 (2006).

24. Suzaki, T., Yoshida, A. \& Hirano, H.Y. Functional diversification of CLAVATA3-related CLE proteins in meristem maintenance in rice. Plant Cell 20, 2049-58 (2008).

25. McCarty, D.R. \& Meeley, R.B. Transposon resources for forward and reverse genetics in maize. in Handbook of Maize: Genetics and Genomics. (eds. Bennetzen, J.L. \& Hake, S.C.) 561-584 (Springer Press, 2009).

26. Yadav, R.K. et al. Plant stem cell maintenance involves direct transcriptional repression of differentiation program. Mol Syst Biol 9, 654 (2013).

27. Chickarmane, V.S., Gordon, S.P., Tarr, P.T., Heisler, M.G. \& Meyerowitz, E.M. Cytokinin signaling as a positional cue for patterning the apical-basal axis of the growing Arabidopsis shoot meristem. Proc. Natl. Acad. Sci. U.S.A. 109, 4002-7 (2012).

28. Gruel, J. et al. An epidermis-driven mechanism positions and scales stem cell niches in plants. Sci. Adv. 2, e1500989 (2016).

29. Kinoshita, A. et al. RPK2 is an essential receptor-like kinase that transmits the CLV3 signal in Arabidopsis. Development 137, 3911-20 (2010).

30. Wu, Q., Luo, A., Zadrozny, T., Sylvester, A. \& Jackson, D. Fluorescent protein marker lines in maize: generation and applications. Int J Dev Biol 57, 535-43 (2013).

31. Clark, S.E., Running, M.P. \& Meyerowitz, E.M. Clavata1, a Regulator of Meristem and Flower Development in Arabidopsis. Development 119, 397-418 (1993).

32. Clark, S.E., Running, M.P. \& Meyerowitz, E.M. Clavata3 Is a Specific Regulator of Shoot and Floral Meristem Development Affecting the Same Processes as Clavata1. Development 121, 2057-2067 (1995).

33. Kayes, J.M. \& Clark, S.E. CLAVATA2, a regulator of meristem and organ development in Arabidopsis. Development 125, 3843-3851 (1998).

34. Bommert, P., Nagasawa, N.S. \& Jackson, D. Quantitative variation in maize kernel row number is controlled by the FASCIATED EAR2 locus. Nat Genet 45, 334-7 (2013).

35. Hsu, Y.C., Li, L. \& Fuchs, E. Transit-amplifying cells orchestrate stem cell activity and tissue regeneration. Cell 157, 935-49 (2014). 


\section{Figure Legends}

Figure 1 fea 3 mutant phenotypes and gene identification. (a) Cleared SAMs of wild type (WT); fea 3 shows increased diameter (arrows) (b). (c) SEM image of wild type tassel primordium (inflorescence meristem shaded yellow); fea 3 tassel shows enlarged inflorescence meristem (d). (e) Wild type tassel (left); fea 3 tassel (right) has a thicker appearance. (f,g) Side and top-down SEM views of wild type ear primordia. (h,i) fea 3 ear primordia, showing enlargement and fasciation of inflorescence meristems. (j) Wild type ear (left) shows regular kernel rows; fea 3 ear (right) shows fasciation and irregular rows. (k) Map-based cloning of fea3, numbers of recombinants/F2 individuals indicated. (l) FEA3 is a predicted receptor like protein with a signal peptide (SP) followed by 12 LRRs, and a transmembrane domain (TM). The position of the fea3-0 insertion and the EMS alleles are shown. Scale bars $\mathrm{a}, \mathrm{b}=100 \mu \mathrm{m}$, others $=500 \mu \mathrm{m}$.

Figure 2 Expression of FEA3 and ZmWUS1. (a) in situ hybridization of FEA3 shows expression in lower layers (predicted organizing center, square bracket) of the vegetative SAM and below it. (b) In the inflorescence apex, FEA3-RFP (red) is expressed similarly. Note that the diffuse and speckled red signal in the L1 SAM layer (*) is background auto-fluorescence. FEA3-RFP is also expressed in leaf primordia (inset). An inflorescence transition stage SAM showing $p Z m W U S 1-N L S . R F P$ expression (red) in presumptive organizing center in wild type (c); expression expands downwards in fea 3 (d). (b-d) counterstained (blue) with calcofluor white cell wall marker $(\mathbf{b}-\mathbf{d}=$ confocal microscopy). All scale bars $=50 \mu \mathrm{m}$.

Figure 3 Interactions between FEA3 and its putative ligand, ZmFCP1. (a,b) fea 3 embryos cultured with control, scrambled peptide (sCLV3) or ZmFCP1 or ESR2c or ZmCLE7 or ZmCLE14. Wild type SAM growth was strongly inhibited by all peptides except sCLV3, and fea 3 was insensitive only to ZmFCP1. (c) in situ hybridization of ZmFCP1, expression was specific to primordia (arrowed). (d) Zmfcp1 mutant; red arrow shows position of $M u$ transposon insertion downstream of the ATG in the first exon. Zmfcpl mutants have a fasciated ear phenotype, seen in top-down SEM view. (e) Cleared SAMs of wild type, $f e a 3$ and $Z m f c p 1$; SAMs of $f e a 3$ and $Z m f c p 1$ were significantly wider (arrowed), but double mutants were not significantly different from fea3 (f). Scale bars $\mathrm{d}=500 \mu \mathrm{m}$, others $=$ $50 \mu \mathrm{m} . \mathrm{n}=20$ for each genotype, mean values \pm s.d. are plotted; ${ }^{* *} P$ value $<0.001$ by two-tailed, twosample $t$ tests; NS $=$ not significant.

Figure 4 Computational model and test of FEA3 function. (a) Computational model predicting effect of genetic perturbations on a hypothetical $Z m C L V 3$ or $Z m W U S 1$ expression, and feedback upregulation of $Z m C L V 3$, see text for details. The model predictions are tested as follows: fea 2 and fea 3 act synergistically in double mutants, in ear meristems (upper images, SEM) and tassels (lower images) (b), and quantified by tassel spikelet density (c). (d) Trans-activated overexpression of $\mathrm{ZmFCP1}$ in primordia led to severe SAM size reduction (e-g). Scale bars $\mathrm{b}=500 \mu \mathrm{m}$, others $=50 \mu \mathrm{m}$. $\mathrm{n}=20(\mathbf{c})$ and $15(\mathrm{~g})$, mean values \pm s.d. are plotted; $* * P$ value $<0.001$ by two-tailed, two-sample $t$ tests; $\mathrm{NS}=$ not significant.

Figure 5 Arabidopsis FEA3 mutants are fasciated. (a) Wild type Landsberg erecta (Ler) plant and SEM image of SAM (shaded, d). (b,c) AtFEA3 RNAi transgenic plants were fasciated, with split inflorescence stems (arrowed), and SEM image of enlarged SAMs (shaded, e). (f) Root growth was strongly inhibited by CLE peptides, except sCLV3 control, but AtFEA3 RNAi lines showed resistance to CLE27 (root tips marked by white lines). (g) Wild type SAM growth was significantly inhibited by CLE27 peptide treatment, but AtFEA3 RNAi plants were resistant to CLE27. $\mathrm{n}=20$ (f) and 19 (g), mean values \pm s.d. are plotted; $* * P$ value $<0.001$ by two-tailed, two-sample $t$ tests; NS $=$ not significant. Scale bars $\mathbf{d}, \mathbf{g}=50 \mu \mathrm{m}, \mathbf{e}=200 \mu \mathrm{m}$.

Figure 6 Yield traits of $f e a 3$ weak alleles. (a) fea 3 weak alleles hybrids had longer and wider ears than normal hybrids, and an increase in kernel row number $(\mathbf{b}, \mathbf{c}$, kernel rows numbered in $\mathrm{b}$ and quantified in c). (d,e) kernel number per ear and ear weight were also significantly higher in fea3-2 or 
fea3-3 hybrids, compared to normal hybrids. $\mathrm{n}=20$, mean values \pm s.d. are plotted; ${ }^{* *} P$ value $<0.001$ by two-tailed, two-sample $t$ tests; NS = not significant. Greek letters indicate significant differences, bars sharing the same letter are not significantly different. 


\section{ONLINE METHODS}

Plant growth and map based cloning. Maize plants were grown in the field or in the greenhouse. The original fea 3 allele, fea3-0, in an unknown genetic background, was crossed to the B73 inbred and selfed to make the F2 population for bulked segregant mapping. 1,000 mutants from the segregating F2 population were used for map-based cloning using molecular markers available from MaizeGDB (fea3-0 genotype data from F2 mapping population are provided in Supplementary data set 1). Phenotyping used the fea3-0 allele, backcrossed 7 times to the $\mathrm{B} 73$ inbred line. Additional alleles were identified using targeted EMS mutagenesis. To measure meristem size, segregating siblings were genotyped and shoot apical meristems of 14-day-old plants (Fig. 1a, b and Fig. 4e, f) or 28-day-old plants (Fig. 3e) were dissected, cleared and measured as described previously ${ }^{10}$. All measurements for all experiments described in this manuscript included at least 10 samples of each genotype, and two or three independent biological replicates, and mean values $+/$ - s.d. were presented, with significance calculated using two-tailed, two-sample $t$ tests, and significant differences reported as $P$ values.

Meristem imaging and in situ hybridization. Scanning electron microscopy was performed on fresh tissues of maize and fixed tissues of Arabidopsis using a Hitachi S-3500N SEM, as described ${ }^{10}$. In situ hybridization experiments were performed as described ${ }^{14}$. Antisense RNAs of FEA3 and ZmFCPI were transcribed and used as probes. Primers are listed in Supplementary Table 2.

Transgene construction and imaging. The FEA3-RFP transgene was constructed by amplification of genomic fragments and fusing the RFP gene in-frame after the signal peptide, or at the C-terminal position, and transformed into maize. We used the MultiSite Gateway System (Invitrogen). All fragments were amplified using PhusionTaq polymerase (Finnzymes) and transferred to the pTF101 Gateway compatible maize transformation vector by the multisite LR recombination. Confirmed clones were transferred to Agrobacterium and transformed into maize, as described ${ }^{36}$. Primers are 
listed in Supplementary Table 2. To determine whether the FEA3-RFP construct could complement the fea3-0 allele, primary FEA3-RFP transformants were crossed to homozygous fea3-0 mutant plants, and F1 plants that carried the FEA3-RFP transgene were backcrossed to homozygous fea3-0 mutants. Transgenic plants of these families were randomly sib-crossed and resulting families subsequently analyzed for complementation. For genotyping, a $2.14 \mathrm{~kb}$ fragment of the fea3-0 locus was amplified; the equivalent amplicon was $1.96 \mathrm{~kb}$ fragment in wild type. The $1.96 \mathrm{~kb}$ PCR fragment also contained the site of RFP insertion, which allowed for simultaneous PCR detection of the transgene. The presence of the transgene locus was additionally monitored by Basta-treatment of leaf tips. The ZmWUS1 domain marker was developed by sub-cloninig 1,576bp upstream of the GRMZM2G047448 gene ATG and 1125 bp downstream of the stop codon into the MultiSite Gateway System (Invitrogen). For nuclear localized fluorescent marker, four repeats of the Vir-E2 NLS sequence ${ }^{37}$ were cloned in frame in front of the $\mathrm{mRFP}^{38}$ and subsequently sub-cloned into the MultiSite Gateway System. Primers are listed in Supplementary Table 2.

For confocal microscopy, tissues were dissected, counterstained with calcoflour white according to manufacture instructions (Sigma-Aldrich 18909) for 2 min and imaged with a Zeiss LSM 710 microscope, using $561 \mathrm{~nm}$ laser excitation and 570-620nm emission for RFP and 405nm laser excitation and 450-500 emission for calcoflour-white. Some tissues were treated with $2 \mu \mathrm{M}$ D15 peptide ${ }^{16}$ (peptide is listed in Supplementary Table 3 ) for $2 \mathrm{hr}$ prior to imaging.

Double mutant analysis. Double mutants were constructed by crossing mutants introgressed into B73, followed by selfing or backcrossing to the F1. All plants were subsequently genotyped (Primers are listed in Supplementary Table 2). Plants were scored for spikelet density by counting the number of spikelets in a $2 \mathrm{~cm}$ region of the tassel central spike, after $2 \mathrm{~cm}$ had been removed from the tip. All genotype data for the double mutant analysis are provided in Supplementary data set 1.

Protein extraction and detection. Aqueous two-phase partitioning was performed as described ${ }^{21}$, using extracts from 1-2 cm maize ear primordia. To inhibit endocytosis, ear primordia were sliced into 
$\sim 2 \mathrm{~mm}$ pieces and were treated with $1 \mathrm{mM}$ D15 peptide for $2 \mathrm{hr}$, or a mock treatment with $1 \mathrm{mM}$ randomized 15-mer peptide (peptide is listed in Supplementary Table 3) as a control. Samples from the upper and lower phases, enriched for plasma membrane or endoplasmic reticulum, respectively, were subjected to SDS-PAGE and western blotting. Blots were probed with antisera for RFP (Chromotek 5F8), or BiP lumenal binding protein (Agrisera AS09-481), or plasma membrane $\mathrm{H}+$ ATPase (Agrisera AS07-260), and detected using a secondary HRP coupled antibody (GE), according to the manufacturer's instructions. This experiment was performed with 2 independent biological replicates.

Phylogenetic analysis. Amino acid sequences of CLE proteins from A. thaliana, maize, and rice were aligned using MUSCLE and the resulting alignment transferred to DNA ${ }^{39}$. Only a very short region of the CLE proteins could be aligned with confidence, thus the final alignment included only the functional ligand (Supplementary Table 3) plus two amino acids on either side. The DNA alignment (Supplementary data set 2 ) was partitioned into $1^{\text {st }}, 2^{\text {nd }}$ and $3^{\text {rd }}$ coding positions, and the best-fitting model of sequence evolution and partitioning scheme was identified using PartitionFinder ${ }^{40}$. The alignment was analyzed both in its entirety, and as two smaller alignments, corresponding to two frequently recovered clades. All alignments were analyzed using MrBayes v3.2.4, with model parameters allowed to vary between data partitions ${ }^{41}$. Two independent runs were initiated in each MrBayes analysis, and both were allowed to run for 10,000,000 generations. The independent runs converged (standard deviation of split frequencies $<0.01$ ) in all analyses.

Peptide assays. Maize embryos segregating for $f e a 3$ and wild type were dissected at 10 days after pollination, when the SAM was exposed, and cultured on gel media ${ }^{21}$ containing scrambled peptide (30 $\mu \mathrm{M}$; Genscript) or ZmFCP1 peptide or ESR2c peptide or ZmCLE7 peptide or ZmCLE14 peptide (peptides are listed in Supplementary Table 3). After 10 days, embryos were harvested for genotyping, and fixed in FAA (10\%, formalin, 5\% acetic acid, 45\% ethanol) and cleared in methyl salicylate, and SAMs measured by microscopy. Experiments used at least 10 embryos per genotype, 
and were replicated in triplicate. For measurement of root growth, mature seeds (Supplementary Fig. 4) or maize embryos dissected at four weeks after pollination segregating for the wild type and fea 3 mutant (Supplementary Fig. 6a) or segregating for fea3;fea2 double mutants (Supplementary Fig. 6b) were germinated on gel media overnight. Synchronized germinating embryos were selected and transferred to gel media in glass test tubes containing scrambled peptide (sCLV3; $5 \mu \mathrm{M}$ or $10 \mu \mathrm{M}$, Genscript) or ESR2c peptide or ZmFCP1 peptide or AtCLV3 peptide or ZmCLE7 peptide or CLE20 peptide or CLE40 peptide or ZmCLE21 peptide or ZmCLE23 peptide (peptides are listed in Supplementary Table 3). After 7 days, root length was measured, and experiments were replicated in triplicate.

For measurement of Arabidopsis roots, sterilized seeds were cultured on half MS media containing scrambled peptide (sCLV3; $1 \mu \mathrm{M}$ ) or AtCLV3 peptide or AtCLE25 peptide or AtCLE26 peptide or AtCLE27 peptide or AtCLE45 peptide (peptides are listed in Supplementary Table 3). After 10 days, root length was measured, and the experiment was replicated in triplicate. For the measurement of Arabidopsis SAMs, sterilized seeds were cultured on half MS liquid media, containing CLV3 $(1 \mu \mathrm{M})$ or sCLV3 or CLE27 and the media was renewed every third day. After two weeks, samples were harvested, fixed, cleared, and SAMs measured by microscopy. Peptide assays were completed with biological replicates performed by different investigators, and meristems were measured in a random order, before grouping based on genotype.

Two-components transactivation assay. The $p Y A B B Y 14-L h G 4$ driver line was constructed using a $Z m Y A B B Y 14$ promoter $(2697 \mathrm{bp})^{42}$ to drive LhG4 expression, and and the $p O p-Z m F C P 1$ reporter line used the complete ZmFCPl coding region (925bp). Fragments were cloned using the MultiSite Gateway System (Invitrogen), as shown in Supplementary Fig. $\mathbf{8 a}^{43}$. Driver and reporter line heterozygotes were crossed to each other and the two-component segregating families were analyzed. Primers are listed in Supplementary Table 2. For real time qPCR, dissected shoot apices $(\sim 2 \mathrm{x} 2$ $\mathrm{x} 3 \mathrm{~mm}$ ) including SAM and young leaves were used. 
Computational methods. The model includes the CLV3/WUS feedback loop and is a development from recent work for A. thaliana $a^{26,28,44}$. It is fully described in the Supplementary Note.

In this system, WUS expression is activated by cytokinin, produced in the external cell layer ${ }^{27}$, upon binding the AHK receptors ${ }^{27,45}$. The CLV3 peptide expressed in the stem cell niche represses WUS expression upon binding its receptor ${ }^{46}$; its own expression is activated by the diffusing transcription factor WUS ${ }^{44}$.

The expression pattern of ZmWUS1 expression in fea3 (Fig. 2d) inside the meristematic tissue, and away from the epidermis, offers insights into its regulation. Interestingly, this expression pattern mirrors the expression pattern of $A H K 4$ in $A$. thaliana ${ }^{27}$, suggesting that a similar cytokinin receptor could be present in maize, underlying ZmWUS1 activation. In the following we will consider the hypothetical expression pattern of ZmAHK4 to be the inner tissue of the SAM. When activated by cytokinin the receptor induces the expression of ZmWUS1 in the model.

As previously shown, the positional information from the diffusion gradient of WUS alone is not sufficient to localise the $C L V 3$ expression domain at the tip of the shoot. In the present work, CLV3 is co-activated by a signal originating from the epidermis allowing the gene expression domains of WUS and CLV3 to mimic their wild type (wt) expression patterns as well as those observed in a large set of mutants ${ }^{26,44,47}$.

The model was ported to maize and adapted to include the experimental findings described in the main text. The model aims at understanding the combined CLE-signalling and the fasciation phenotype observed in the fea 3 mutant. The domain of activity of the wt CLV3 promoter is used as a marker for the stem cell domain (central zone), and a large increase of its activity will be considered as leading to a fasciated phenotype ${ }^{48}$.

The tissue template used for the modelling is a three-dimensional representation of the maize SAM (Supplementary Fig. 7). The expression domains of genes of interest are defined from experimental data: ZmWUS1 (Fig. 2c and ${ }^{18}$ ), ZmCLE7 (hereafter referred to as ZmCLV3; phylogeny in 


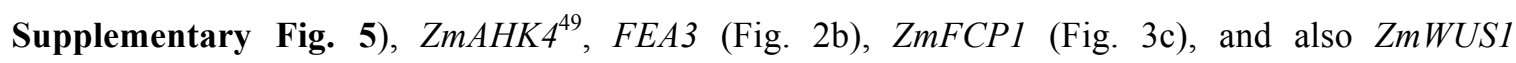
expression in fea3 (Fig. 2d).

A schematic description of the model is shown in Supplementary Fig. 8. ZmWUS1 expression is driven by cytokinin binding the ZmAHK4 receptors. FEA3 receptors upon binding ZmFCP1, and FEA2 receptors upon binding either ZmCLV3 or ZmFCP1 (as supported by peptide assays in Fig 3a and Supplementary Fig. 6b), repress ZmWUS1 expression. FEA2 is constitutively expressed in the model $^{10}$. ZmWUS1 and a hypothetical epidermis-originating signal activate ZmCLV3 expression. Gene expression is modelled using Hill functions and cell-to-cell transport of molecules is passive. The complete model is described by a set of seven differential equations for each cell of the tissue template (Supplementary Note).

The parameter values are inferred via an optimisation strategy adapted from ${ }^{28}$ and making use of the CMA-ES algorithm ${ }^{50}$. Multiple optimisations resulted in 203 parameter sets successfully describing the wt expression of ZmWUS1 and ZmCLV3. The model was further tested for loss of function of ZmCLV3, FEA2, FEA3, FEA3; FEA2, FCP1 and over-expression of FCP1. The variation of expression of both ZmWUS1 and ZmCLV3 in all conditions is presented in Supplementary Fig. 9, 10. fea2, fea3 and fea3; fea2 all lead to a substantial increase of both ZmWUS1 and ZmCLV3, the model thus predicts a fasciated phenotype for the three mutants, which proves to be consistent with the experimental data presented in Fig. 4. Moreover, as shown, the severity of the phenotypes increases from fea 2 to $f e a 3$ to $f e a 3$; fea 2 . Both the fea 3 and fea3; fea2 mutants result in a large downward expansion of the ZmWUS1 domain, similar to the pZmWUS1-NLS.RFP expression domain observed in fea3 (Fig. 2).

fcp 1 causes a large expansion of ZmWUS1 and ZmCLV3 and a predicted fasciated phenotype. The ZmWUS1 domain exhibits a downward expansion typical of the fea 3 mutants.

Finally, over-expressing the $f_{c p} 1$ peptide in its own expression domain (using a primordium specific YABBY promoter) causes a decrease of ZmWUS1 expression, leading to a reduction of ZmCLV3 
expression. The phenotype suggests a smaller stem cell pool and thus a reduction of the meristem size or meristem arrest.

The model described above is largely inspired by data obtained in A. thaliana. In particular, the $L O G$ genes, whose expression patterns are unknown in maize, are expressed in the epidermis; in rice the expression resembles the stem cell domain ${ }^{51}$. In order to assess the importance of this choice we modified the model such that the production of active cytokinin would not be limited to the epidermis, but to the tip of the meristem, as suggested from the data obtained in rice.

This second "rice-like" model was optimised in the same fashion as the previous model, resulting in 295 parameter sets. Strikingly, the model behaves extremely similarly to the "Arabidopsis-like" model (Supplementary Fig. 11, 12), suggesting that the pattern of expression of the LOGs in maize could be similar to either organisms without affecting the conclusions of the present study.

AtFEA3 RNAi and artificial microRNA construction. For RNAi of At3g25670, the full length cDNA, or the 3' untranslated region (289bp) was cloned into the pHellsgate8 (SnapGene). The precursors for artificial miRNAs against At3g25670 (TGCTCTATACATCAATGGAAA and TCGCCGATAAGAATACCTAAA) were cloned into the miR159a backbone following synthesis (Genscript) and then were mobilized into pDONR P4r-P3r. The artificial miRNAs were driven by p35S or an AGAMOUS promoter, $\mathrm{pAGm}^{52}$. We used the MultiSite Gateway System (Invitrogen). All fragments were transferred to a modified pART27 Gateway compatible arabidopsis transformation vector by the multisite LR recombination. Primers are listed in Supplementary Table 2. To measure meristem size, shoot apical meristems of 14-day-old plants were dissected, cleared and measured as described previously ${ }^{10}, \mathrm{n}=16$ (wild type, weak lines) and 8 (strong lines).

Quantification of transcript abundance. Q-RTPCR analysis was performed on a CFX96 Real-time system (Bio-RAD), as described ${ }^{53}$. Total mRNAs were extracted using an RNA extraction kit (Qiagen). The target cycle threshold values were normalized using ZmUbiquitin or AtActin2. Data are presented as the mean of three biological replicates and three technical replicates. Primers are listed in 
the Supplementary Table 2. RNA-seq reads from inflorescence meristem tips were obtained from wild type and fea 3 mutant ear primordia as previously described ${ }^{54}$. Two biological replicates (pools of $\sim 30$ inflorescence meristem tips) were compared between wild type and fea 3 mutants. Reads were mapped to the maize reference genome (AGPv3) using TopHat $2^{55}$ and counts were normalized by the total number of reads mapped before plotting ${ }^{56}$.

Yield trials. fea 3 EMS alleles developed in the non-complementation screen were backcrossed to W22 or B73 2 - 4 times, and F1 homozygous mutants hybrids generated by crossing. Heterozygous fea3-2 or fea3-3 mutants hybrids were used as the control "normal" hybrids. Field tests were performed at Uplands Farm (Cold Spring Harbor, New York) in the summer of 2015. For analysis of yield parameters, 20-30 open-pollinated ears with full seed set were analyzed.

36. Mohanty, A. et al. Advancing Cell Biology and Functional Genomics in Maize Using Fluorescent Protein-Tagged Lines. Plant Physiology 149, 601-605 (2009).

37. Crawford, K.M. \& Zambryski, P.C. Subcellular localization determines the availability of non-targeted proteins to plasmodesmatal transport. Curr Biol 10, 1032-40 (2000).

38. Osterrieder, A. et al. Fluorescence lifetime imaging of interactions between Golgi tethering factors and small GTPases in plants. Traffic 10, 1034-46 (2009).

39. Edgar, R.C. MUSCLE: multiple sequence alignment with high accuracy and high throughput. Nucleic Acids Res 32, 1792-7 (2004).

40. Lanfear, R., Calcott, B., Ho, S.Y. \& Guindon, S. Partitionfinder: combined selection of partitioning schemes and substitution models for phylogenetic analyses. Mol Biol Evol 29, 1695-701 (2012).

41. Ronquist, F. et al. MrBayes 3.2: efficient Bayesian phylogenetic inference and model choice across a large model space. Syst Biol 61, 539-42 (2012).

42. Juarez, M.T., Twigg, R.W. \& Timmermans, M.C. Specification of adaxial cell fate during maize leaf development. Development 131, 4533-44 (2004).

43. Krishnakumar, V. et al. A maize database resource that captures tissue-specific and subcellularlocalized gene expression, via fluorescent tags and confocal imaging (Maize Cell Genomics Database). Plant Cell Physiol. 56, e12(1-7) (2015).

44. Yadav, R.K. et al. WUSCHEL protein movement mediates stem cell homeostasis in the Arabidopsis shoot apex. Genes Dev 25, 2025-30 (2011).

45. Gordon, S.P., Chickarmane, V.S., Ohno, C. \& Meyerowitz, E.M. Multiple feedback loops through cytokinin signaling control stem cell number within the Arabidopsis shoot meristem. Proc. Natl. Acad. Sci. U.S.A. 106, 16529-34 (2009).

46. Ogawa, M., Shinohara, H., Sakagami, Y. \& Matsubayashi, Y. Arabidopsis CLV3 peptide directly binds CLV1 ectodomain. Science 319, 294 (2008).

47. Jönsson, H., Shapiro, B.E., Meyerowitz, E.M. \& Mjolsness, E. Signalling in multicellular models of plant development. in On growth, form and computers, (eds. Sanjeev Kumar and Peter J. Bentley) 156161 (Elsevier Academic Press, 2003).

48. Iliev, I. \& Kitin, P. Origin, morphology, and anatomy of fasciation in plants cultured in vivo and in vitro. Plant Growth Regulation 63, 115-129 (2011).

49. Yonekura-Sakakibara, K., Kojima, M., Yamaya, T. \& Sakakibara, H. Molecular characterization of cytokinin-responsive histidine kinases in maize. Differential ligand preferences and response to ciszeatin. Plant Physiol 134, 1654-61 (2004). 
50. Hansen, N. The CMA Evolution Strategy: A Comparing Review. in Towards a new evolutionary computation. Advances in estimation of distribution algorithms. (eds. Lozano, J.A., Larrañga, P., Inza, I. \& Bengoetxea, E.) 75-102 (Springer Press, 2006).

51. Kurakawa, T. et al. Direct control of shoot meristem activity by a cytokinin-activating enzyme. Nature 445, 652-5 (2007).

52. Hong, R.L., Hamaguchi, L., Busch, M.A. \& Weigel, D. Regulatory elements of the floral homeotic gene AGAMOUS identified by phylogenetic footprinting and shadowing. Plant Cell 15, 1296-309 (2003).

53. Whipple, C.J. et al. grassy tillers1 promotes apical dominance in maize and responds to shade signals in the grasses. Proc. Natl. Acad. Sci. U.S.A. 108, E506-12 (2011).

54. Eveland, A.L. et al. Regulatory modules controlling maize inflorescence architecture. Genome Res 24, 431-43 (2014).

55. Kim, D. et al. TopHat2: accurate alignment of transcriptomes in the presence of insertions, deletions and gene fusions. Genome Biol 14, R36 (2013).

56. Trapnell, C. et al. Differential gene and transcript expression analysis of RNA-seq experiments with TopHat and Cufflinks. Nat Protoc 7, 562-78 (2012). 


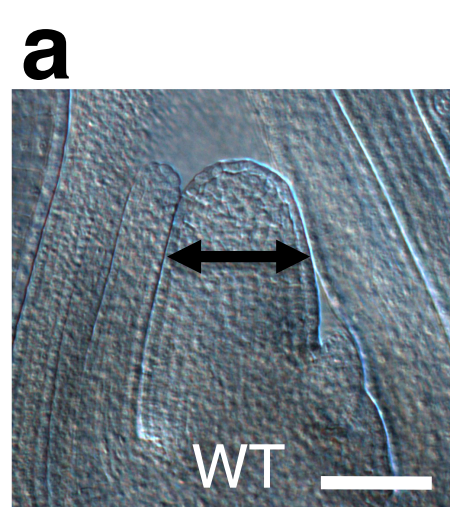

c

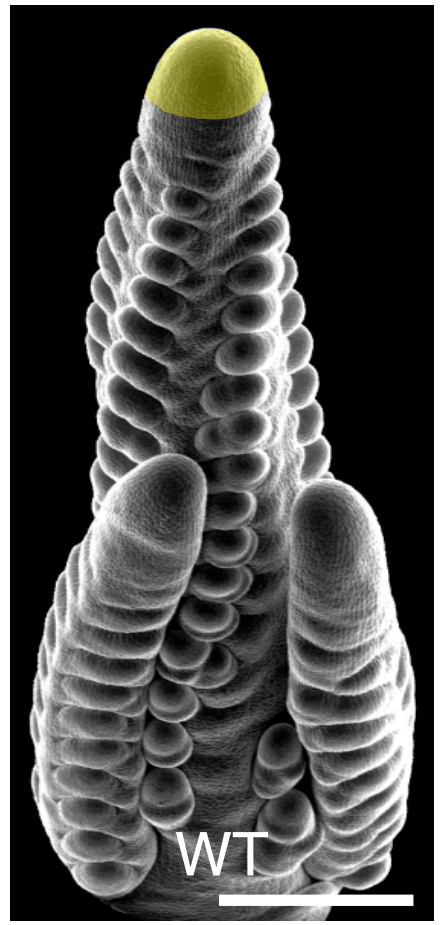

d
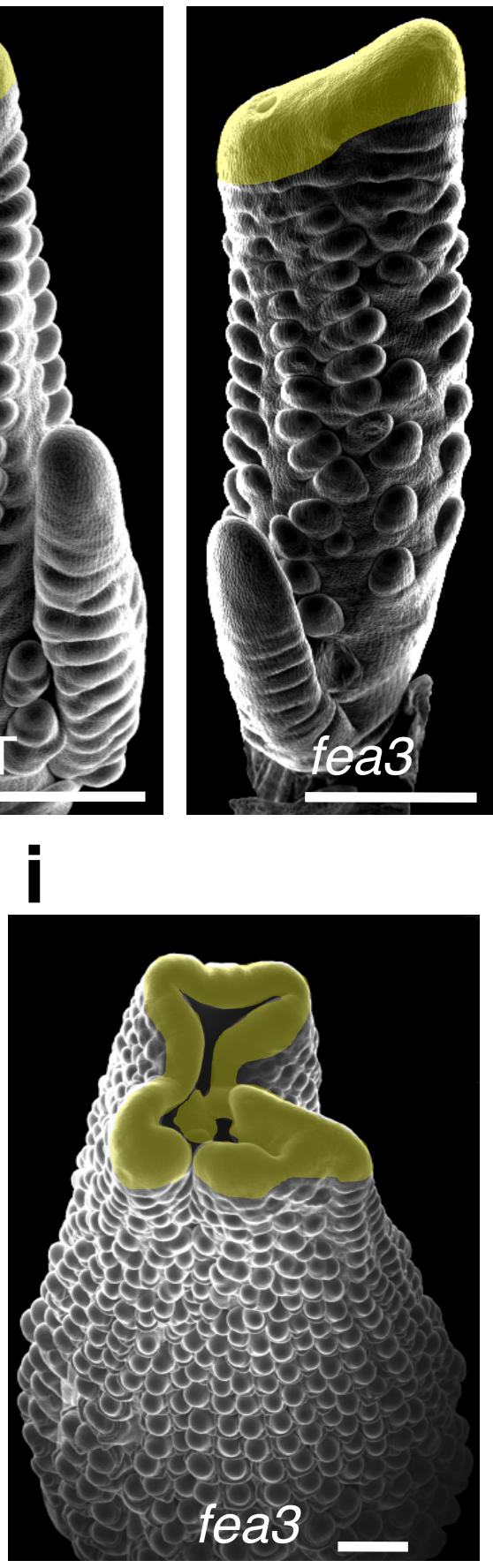

e

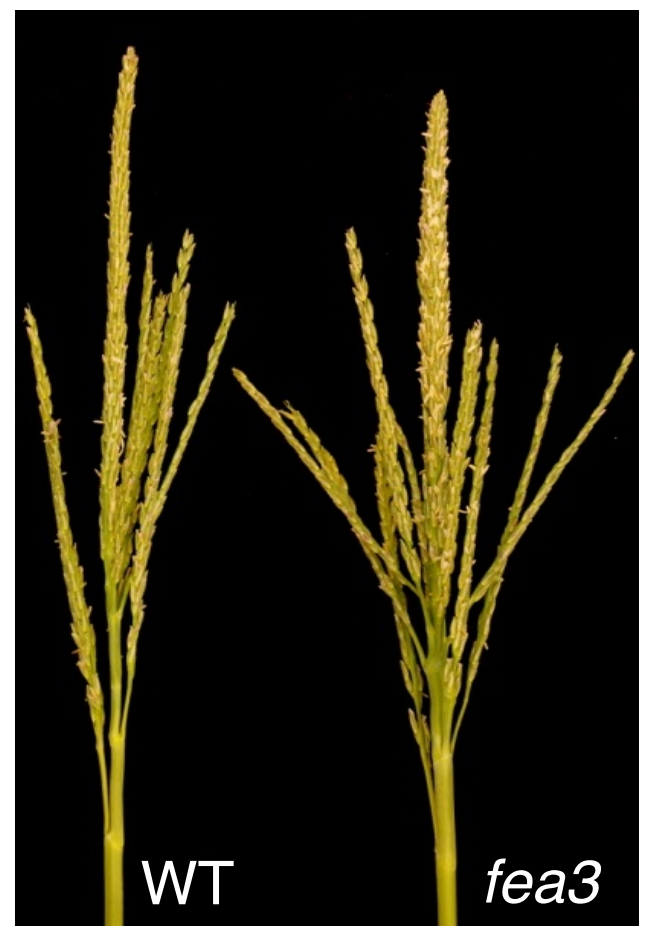

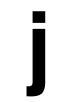

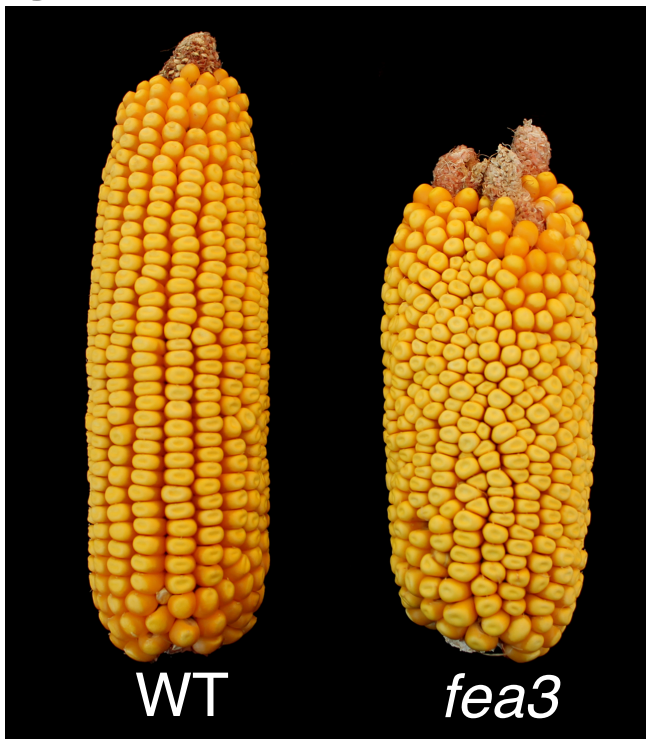

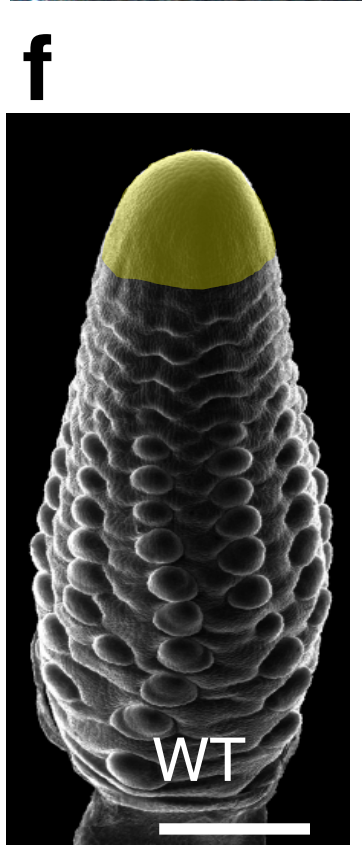
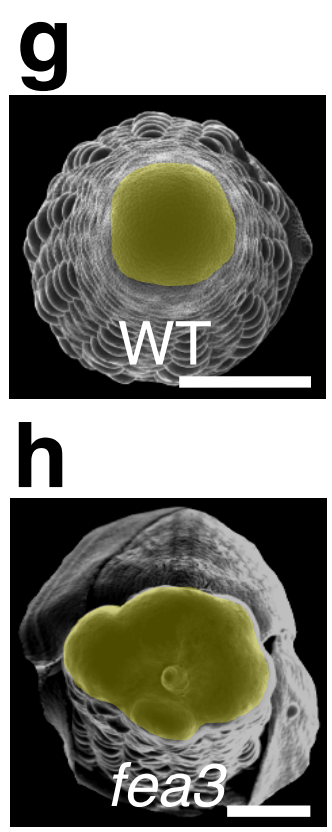

k Maize Chr. 3

$28.9 \mathrm{Mb}$

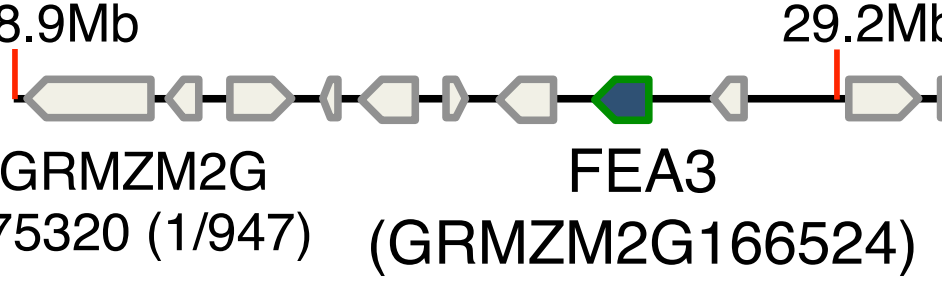

I

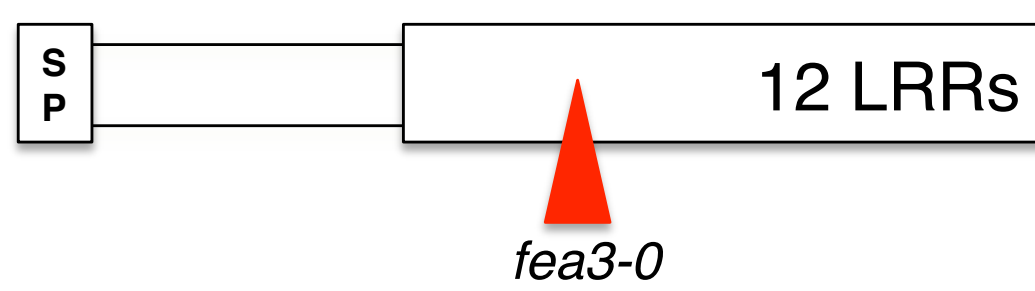

Partial retro-transposon

fea3-1 fea3-2 C435Y

D336N C417Y
29.6Mb

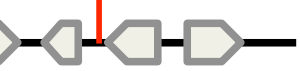

GRMZM2G

$112598(1 / 947)$ 
a

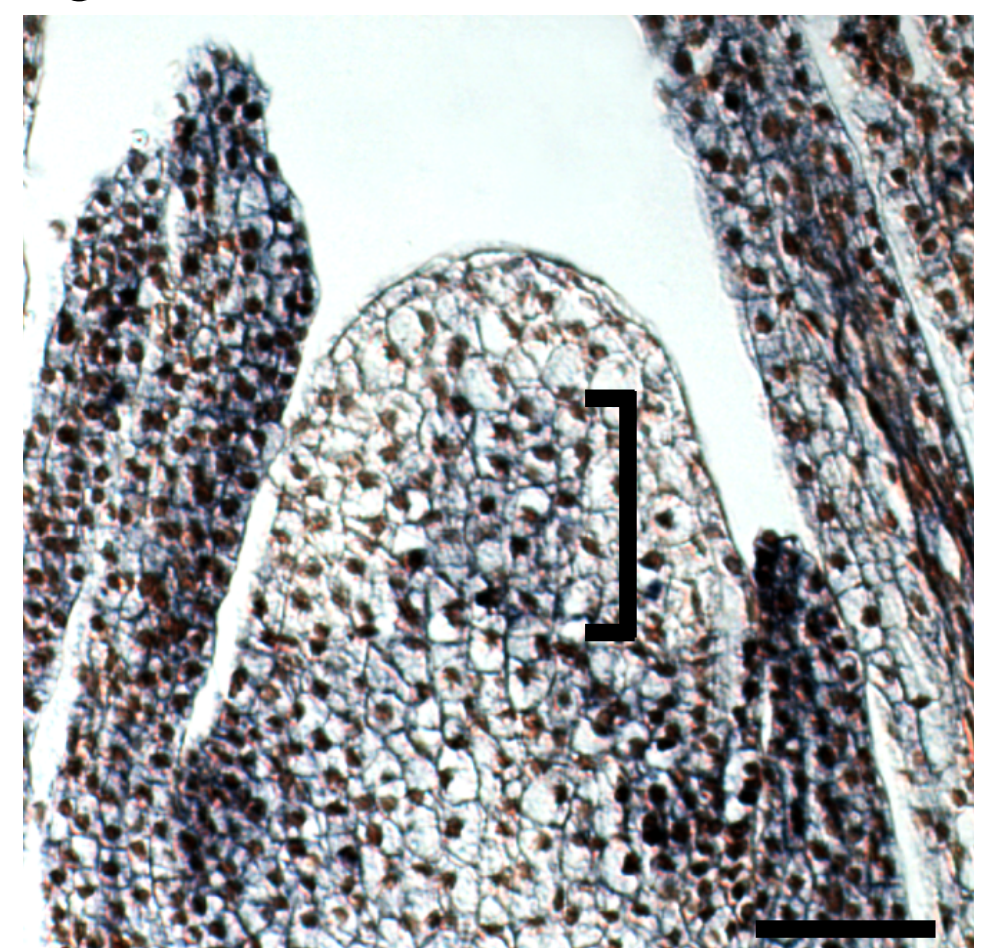

C

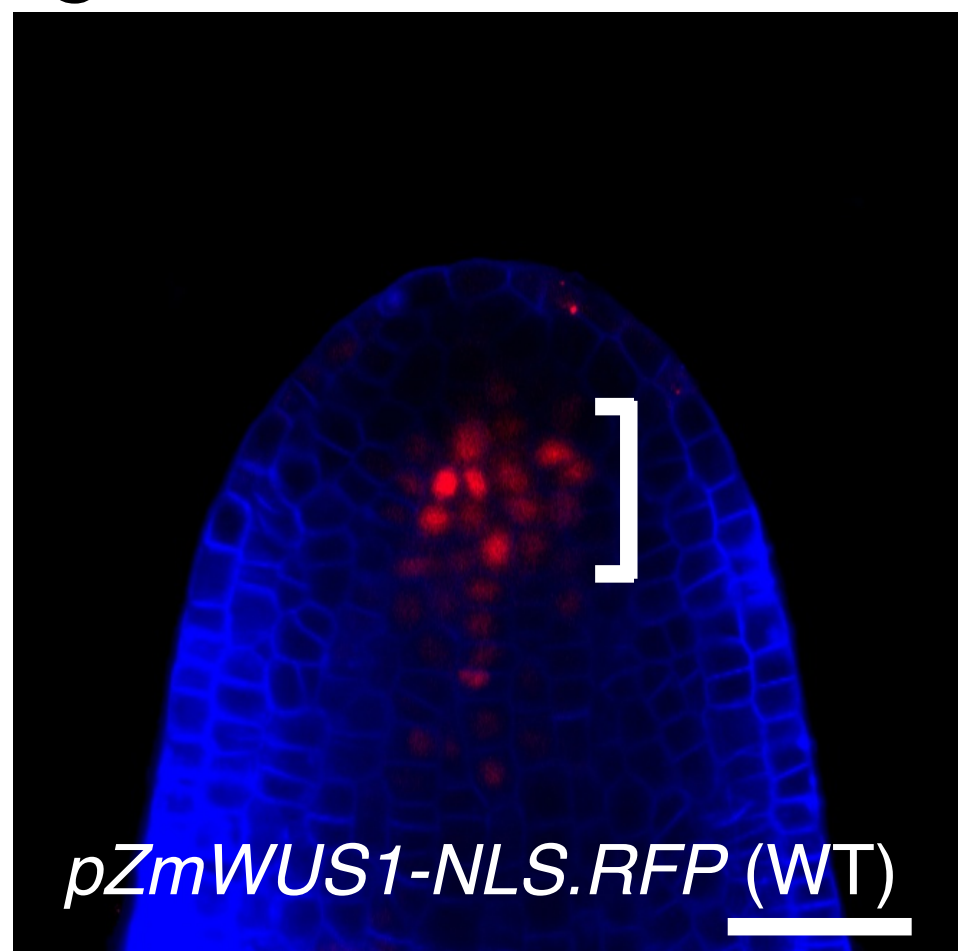

b

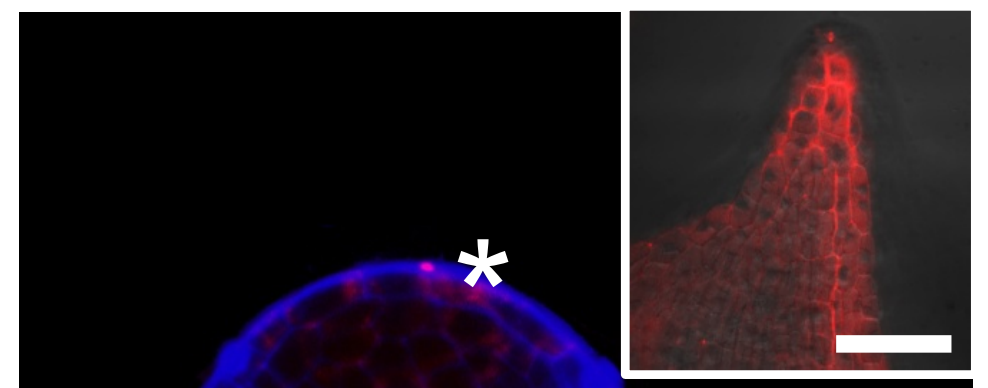

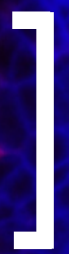

pFEA3-FEA3:RFP

(WT)

\section{d}

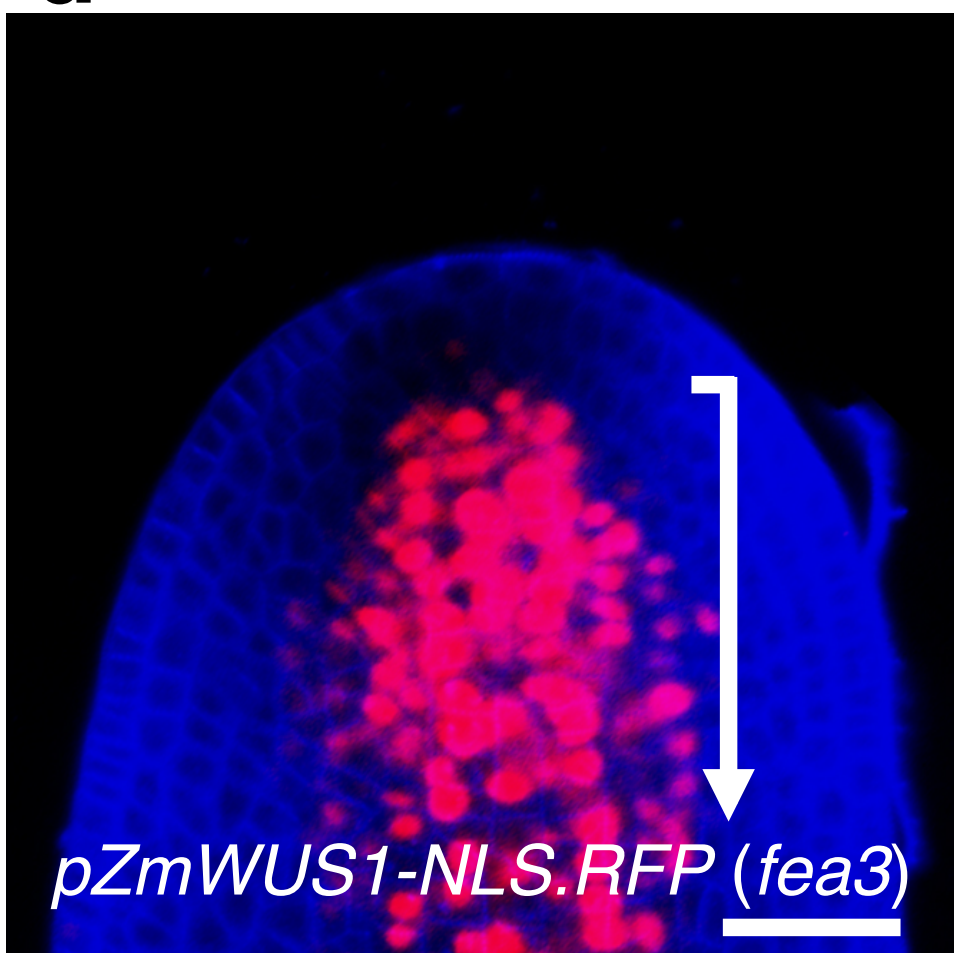



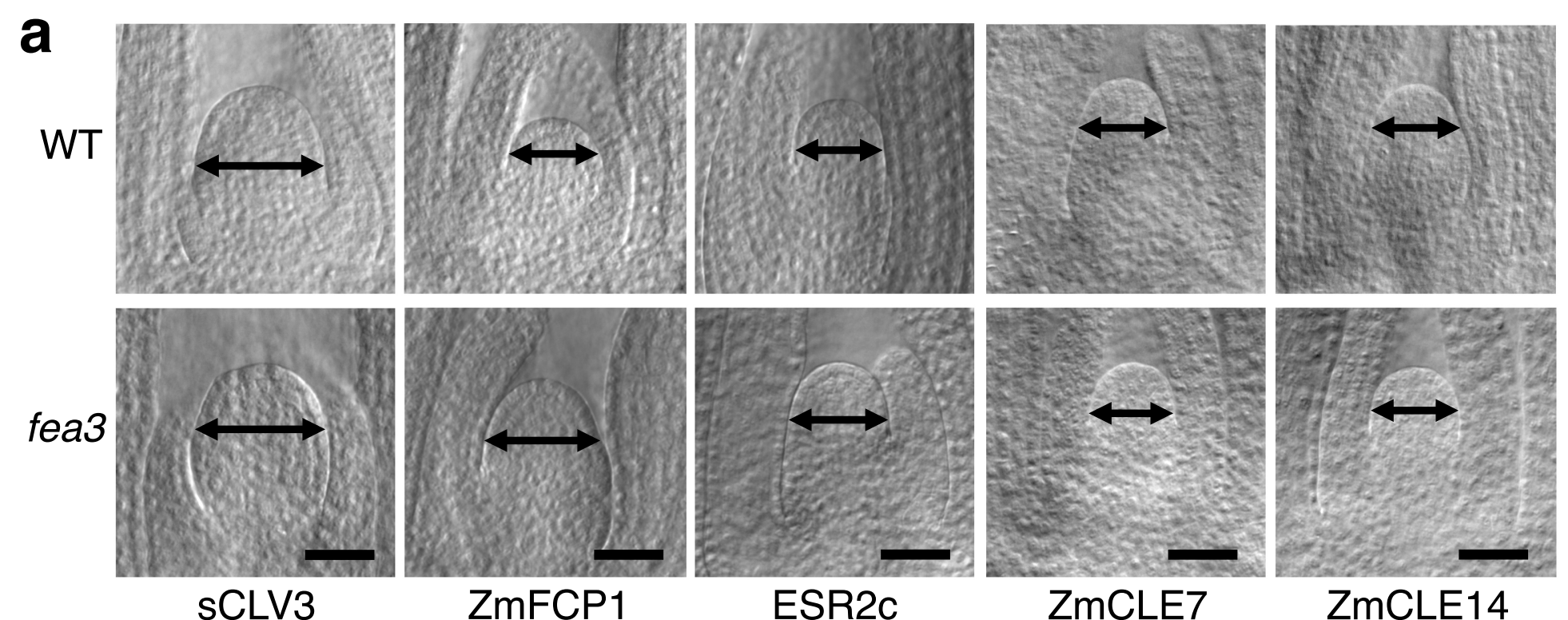

b


f

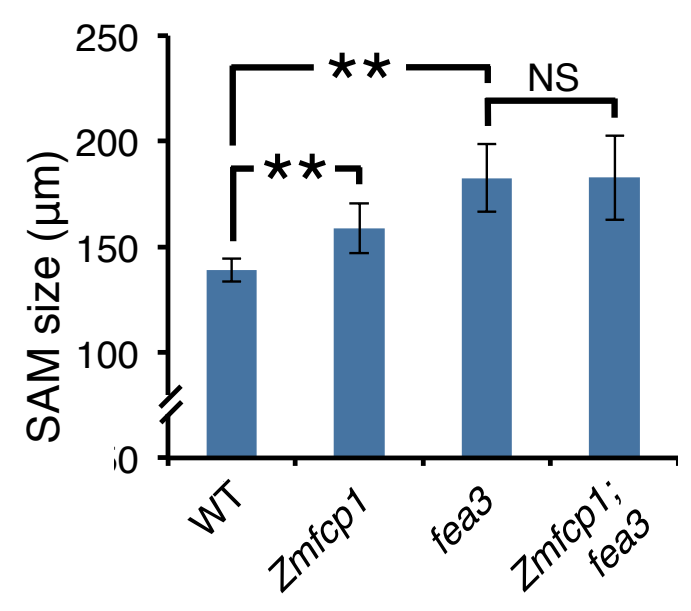




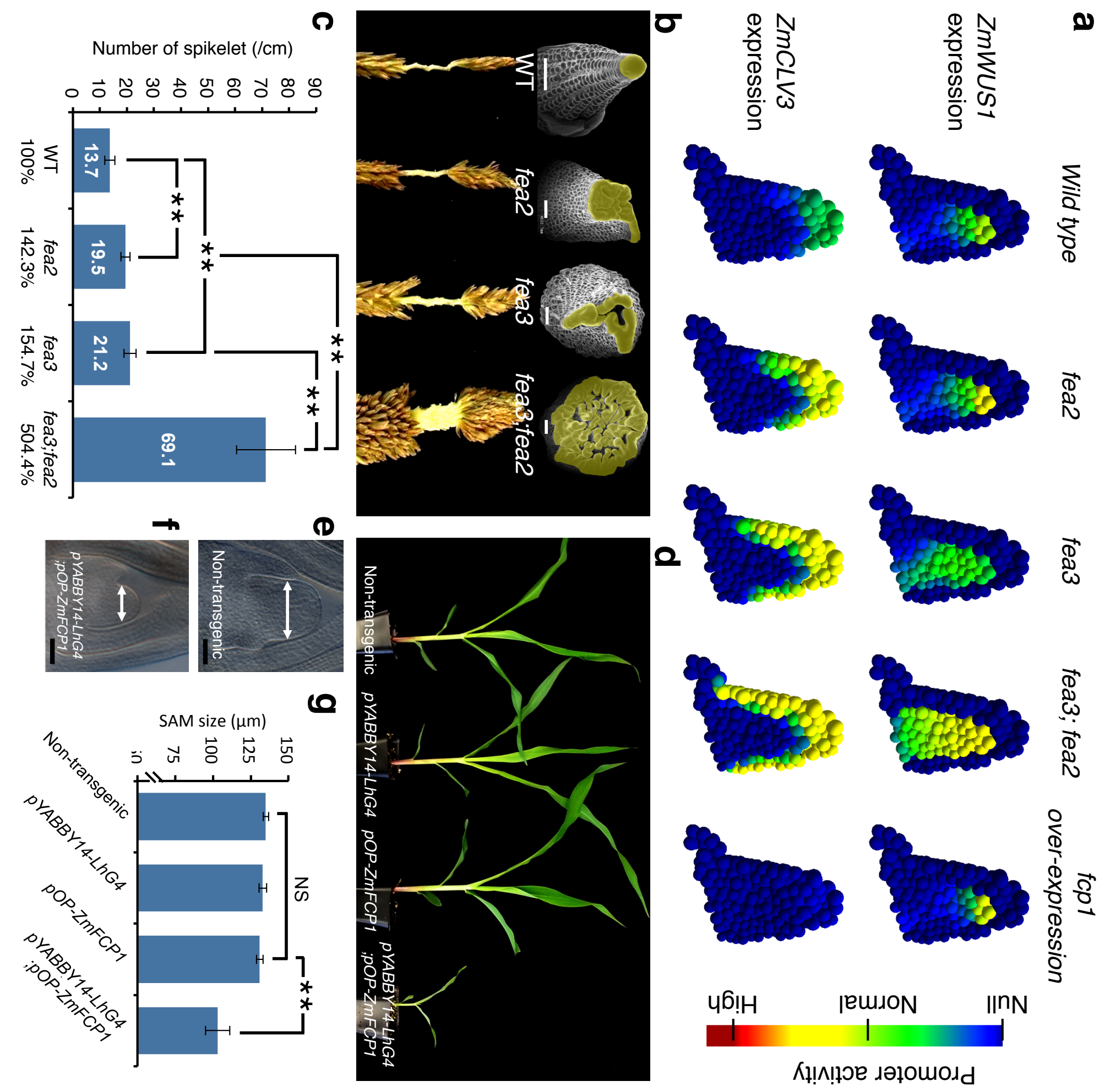



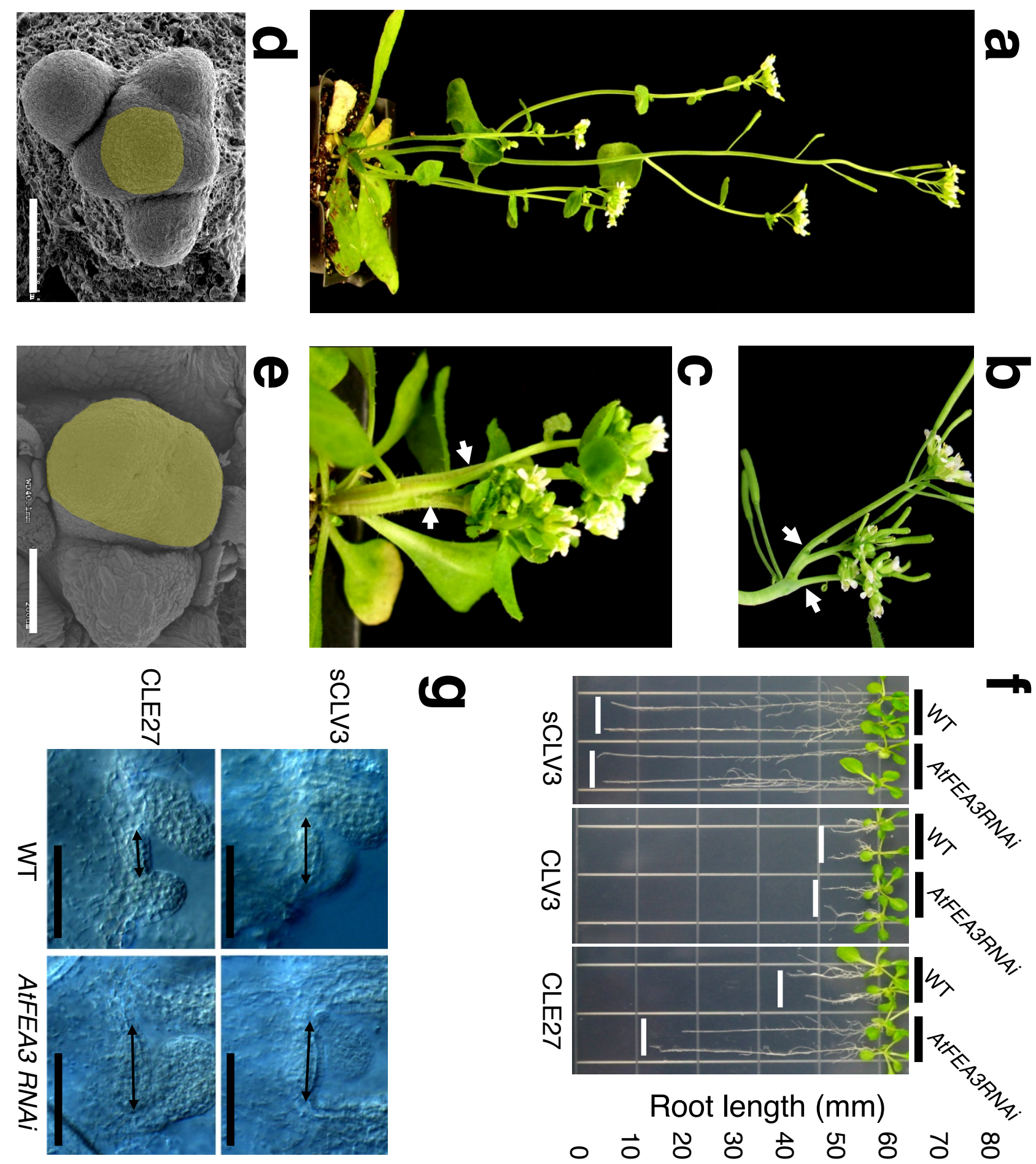

10

SAM size $(\mu \mathrm{m})$
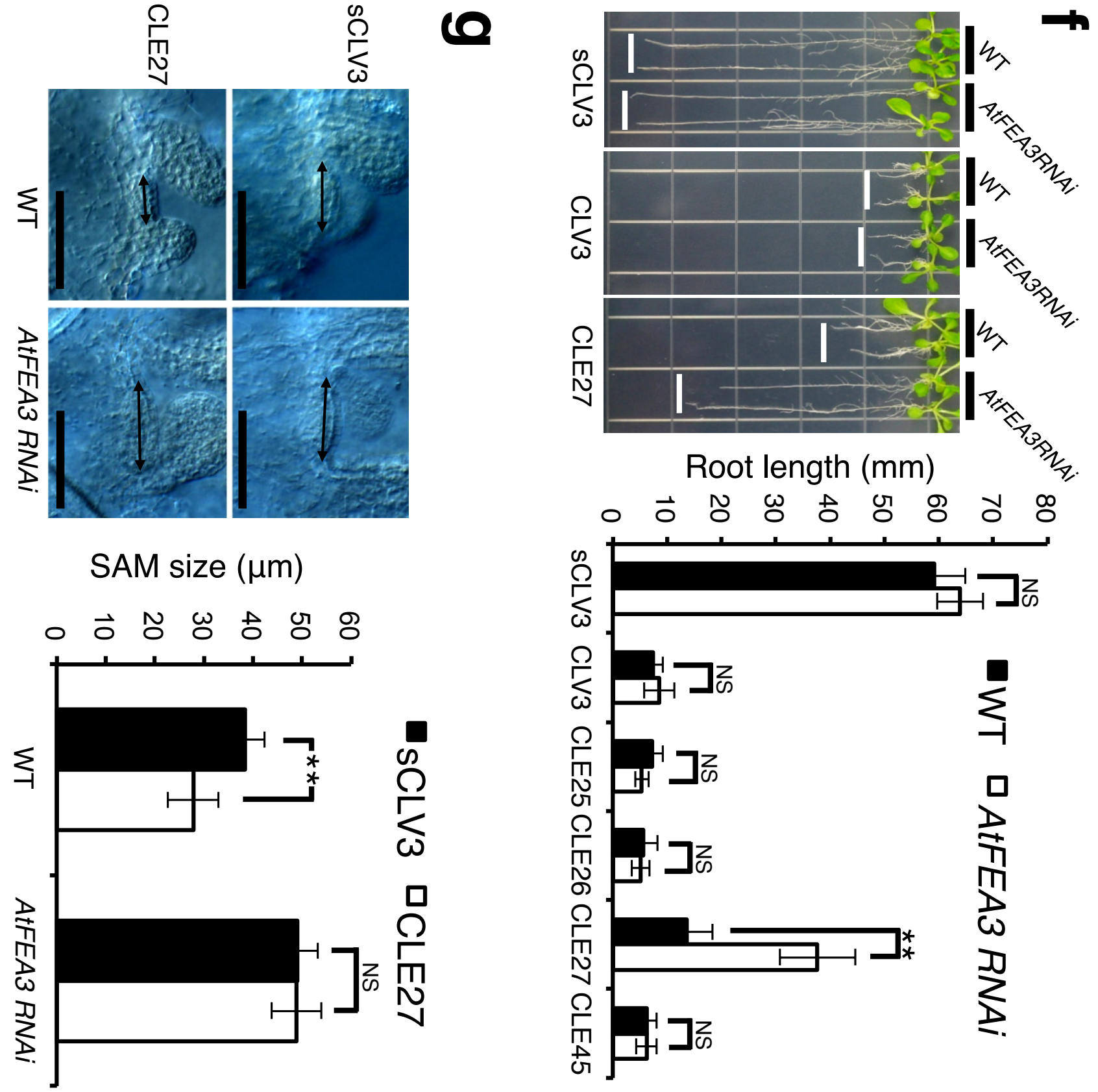


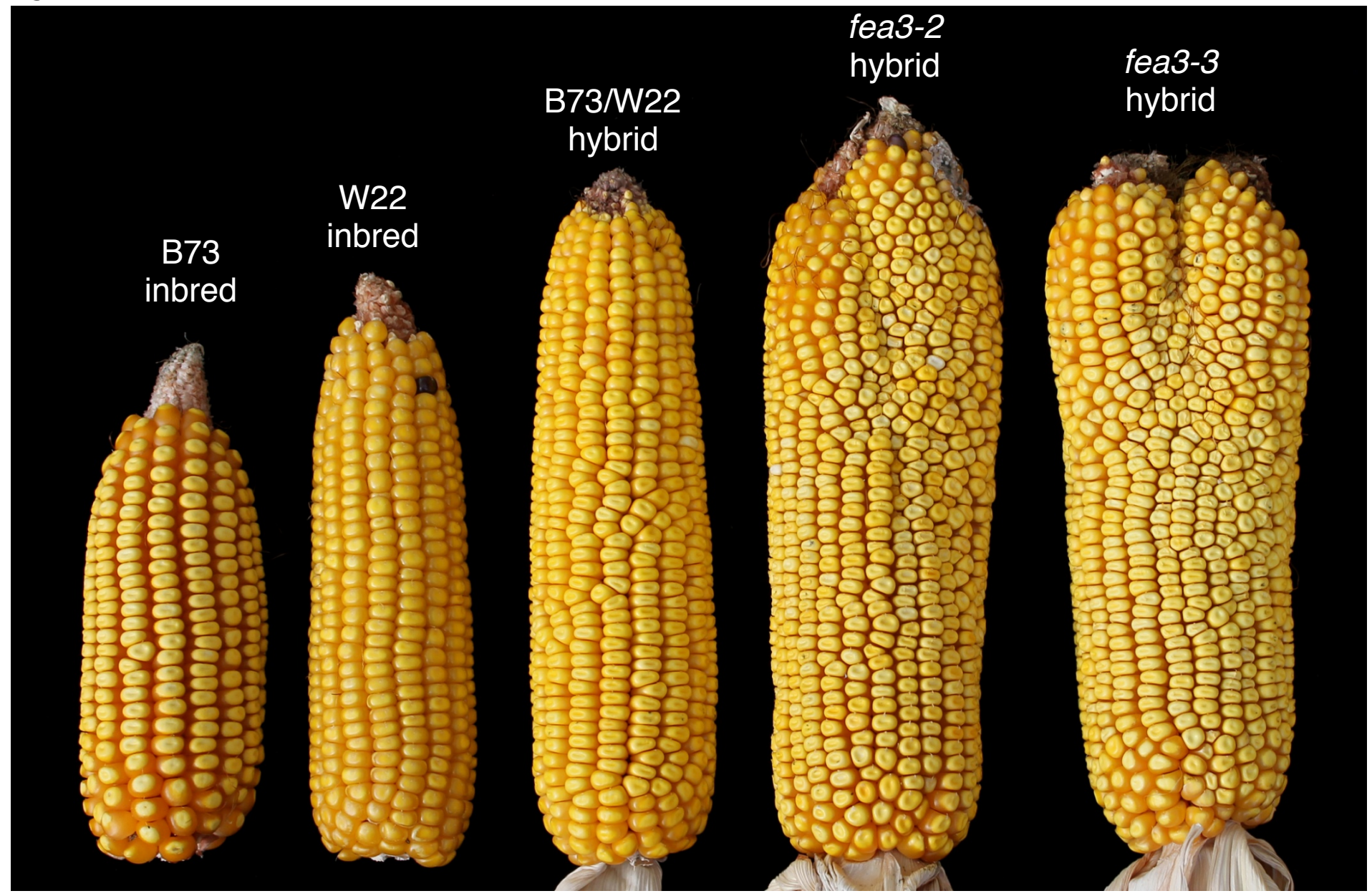

\section{b}

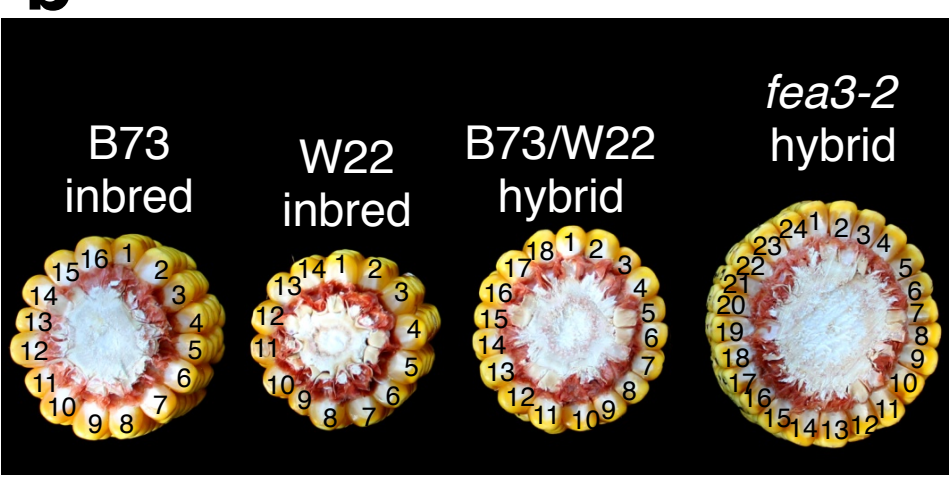

\section{d}

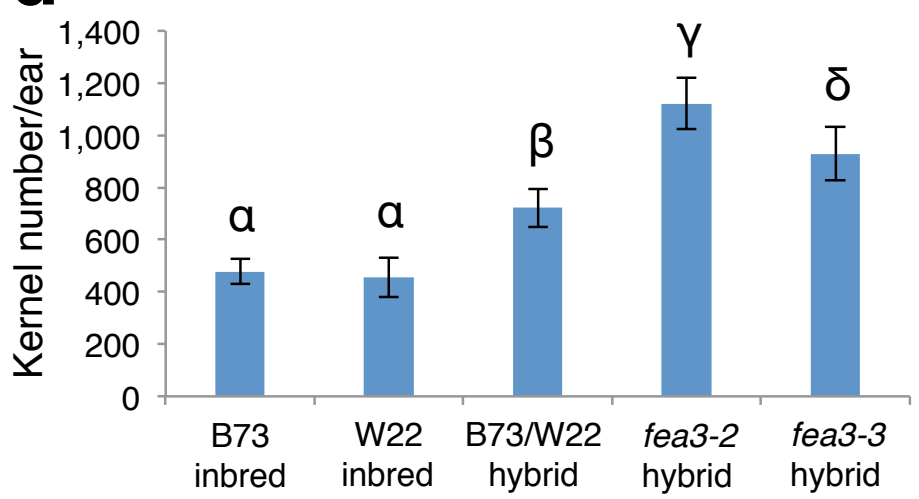

\section{C}

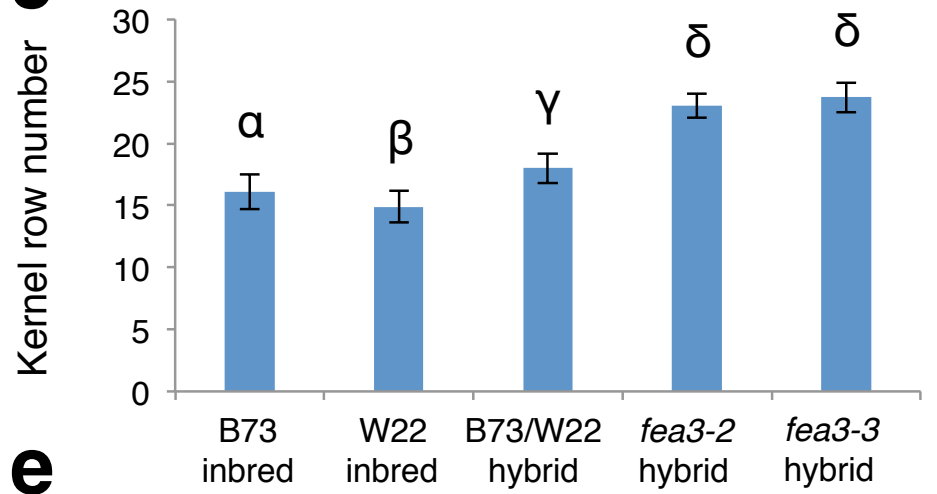

\section{0}

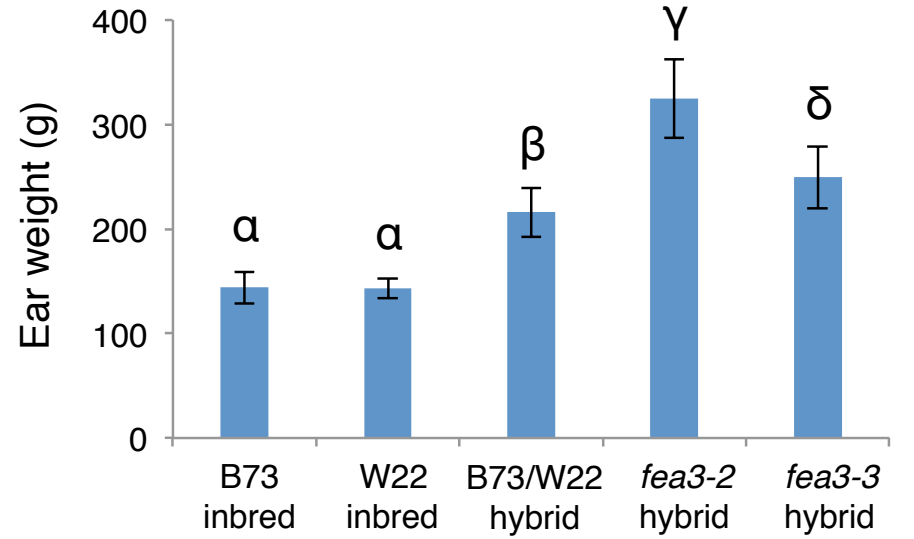


Supplementary Table 1 Summary table of yield traits.

\begin{tabular}{|c|c|c|c|c|c|c|}
\hline & Ear length $(\mathrm{cm})$ & $\begin{array}{c}\text { Ear thickness } \\
(\mathrm{cm})\end{array}$ & $\begin{array}{c}\text { Kernel row } \\
\text { number }\end{array}$ & $\begin{array}{c}\text { Kernel } \\
\text { number/row }\end{array}$ & kernel number/ear & Ear weight $(\mathrm{g})$ \\
\hline $\mathrm{B} 73$ & $15.03 \pm 0.8(\mathrm{a})$ & $4.73 \pm 0.2(\mathrm{a})$ & $16.1 \pm 1.4(\mathrm{a})$ & $29.7 \pm 2.3(\mathrm{a})$ & $478.6 \pm 49(\mathrm{a})$ & $143.8 \pm 15.1(\mathrm{a})$ \\
\hline $\mathrm{W} 22$ & $16.97 \pm 0.9(\beta)$ & $4.4 \pm 0.2(\beta)$ & $14.9 \pm 1.3(\beta)$ & $30.4 \pm 3.1(\mathrm{a})$ & $454.5 \pm 76(\mathrm{a})$ & $142.1 \pm 9.3(\mathrm{a})$ \\
\hline B73/W22 & $19.19 \pm 0.9(\mathrm{\gamma})$ & $4.75 \pm 0.3(\mathrm{a})$ & $18 \pm 1.2(\mathrm{\gamma})$ & $42.4 \pm 3.2(\beta)$ & $721.7 \pm 70(\beta)$ & $215.8 \pm 23.4(\beta)$ \\
\hline $\begin{array}{c}\text { fea3-2 } \\
\text { hybrid }\end{array}$ & $20.29 \pm 0.8(\delta)$ & $5.73 \pm 0.2(\mathrm{\gamma})$ & $23.03 \pm 1(\delta)$ & $45.9 \pm 2.6(\mathrm{\gamma})$ & $1,125.0 \pm 99.4(\mathrm{\gamma})$ & $324.6 \pm 37.9(\mathrm{\gamma})$ \\
\hline $\begin{array}{c}\text { fea3-3 } \\
\text { hybrid }\end{array}$ & $18.54 \pm 0.8(\varepsilon)$ & $5.71 \pm 0.1(\mathrm{\gamma})$ & $23.6 \pm 1.2(\delta)$ & $41.1 \pm 3.6(\beta)$ & $929.0 \pm 103(\delta)$ & $249.2 \pm 29.5(\delta)$ \\
\hline
\end{tabular}

$P$ value $<0.001$, errors represent s.d.; $n=20-30$, values sharing the same letter in rows are not significantly different. 
Supplementary Note Computational model.

The model includes the $C L V 3 / W U S$ feedback loop and is a development from recent work for $A$. thaliana ${ }^{26,28,44}$. In this system WUS expression is activated by cytokinin, produced in the external cell layer $^{27}$, upon binding the AHK receptors ${ }^{27,45}$. The CLV3 peptide expressed in the stem cell niche represses WUS expression upon binding its receptor ${ }^{46}$; its own expression is activated by the diffusing transcription factor $\mathrm{WUS}^{44}$.

The expression pattern of ZmWUS1 expression in fea3 (Fig. 2d) inside the meristematic tissue, and away from the epidermis, offers insights into its regulation. Interestingly this expression pattern mirrors the expression pattern of $A H K 4$ in $A$. thaliana ${ }^{27}$, suggesting that a similar cytokinin receptor could be present in maize, underlying ZmWUS1 activation. In the following we will consider the hypothetical expression pattern of $Z m A H K 4$ to be the inner tissue of the SAM. When activated by cytokinin the receptor induces the expression of ZmWUS1 in the model.

As previously shown, the positional information from the diffusion gradient of WUS alone is not sufficient to localise the $C L V 3$ expression domain at the tip of the shoot. In the present work, $C L V 3$ is co-activated by a signal originating from the epidermis allowing the gene expression domains of WUS and CLV3 to mimic their wild type expression patterns as well as those observed in a large set of mutants ${ }^{26,44,47}$

The model was ported to maize and adapted to include the experimental findings described in the main text. The model aims at understanding the combined CLE-signalling and the fasciation phenotype observed in the fea3 mutant. The domain of activity of the wild type CLV3 promoter is used as a marker for the stem cell domain (central zone), and a large increase of its activity will be considered as leading to a fasciated phenotype ${ }^{48}$.

\section{Meristem tissue template}

The SAM is represented by a set of spherical cells. Overlapping spheres are considered as cells in contact and can exchange diffusing molecules. The complete tissue counts 806 cells representing the shape of a maize meristem including two primordia (Supplementary Fig. 7). The expression domains 
of genes of interest are defined from experimental data: wild type expression for ZmWUS1 (Fig. 2c and ${ }^{18}$ ), ZmCLE7 (hereafter referred to as ZmCLV3; phylogeny in Supplementary Fig. 5), ZmAHK $4^{49}$, FEA3 (Fig. 2b), ZmFCP1 (Fig. 3c), and also ZmWUS1 expression in fea3 (Fig. 2d). The domains of ZmAHK4 and ZmCLV3 are adapted from A. thaliana expression domains in the SAM. Two types of boundaries are defined on the system: (i) the bottom boundary, $B$, abstracting the interface with the tissue below the meristem and where diffusing molecules are removed from simulations based on their diffusion rate, and (ii) the cells of the epidermis $(L 1)$ (Supplementary Fig. 7).

\section{Gene expression}

RNA production is modelled using Hill functions; RNAs undergo a degradation $g$. For a set of $N_{A}$ activators $\left\{A_{a}\right\}$ and $N_{I}$ inhibitors $\left\{I_{b}\right\}$, the concentration of a RNA, $X$, varies as:

$$
\frac{d X}{d t}=V \prod_{A_{a}}^{N_{A}} \frac{A_{a}^{n}}{A_{a}^{n}+k_{a}^{n}} \prod_{I_{b}}^{N_{I}} \frac{k_{b}^{n}}{I_{b}^{n}+k_{b}^{n}}-g X
$$

with $V$ the maximal rate of RNA production. The Hill constants, $k$, set the required concentration of activators or inhibitors to switch a gene between its active and inactive states. The Hill coefficients, $n$, control the slope of the transition between states. The equilibrium of $X$ is found for:

$$
X=\frac{V \prod_{A_{a}}^{N_{A}} \frac{A_{a}^{n}}{A_{a}^{n}+k_{a}^{n}} \prod_{I_{b}}^{N_{I}} \frac{k_{b}^{n}}{I_{b}^{n}+k_{b}^{n}}}{g}
$$

\section{Diffusing molecules}

Diffusing molecules are produced within a gene expression domain (ZmWUS1, ZmCLV3, ZmFCP1) or in the L1. This domain is referred to as $P$, a vector of cell RNA concentrations for gene expression or a cell vector of 1 or 0 indicating that a cell belongs to the $\mathrm{L} 1$ or not. The domain is associated to a production rate $p$. Similar to the L1, $B$ is a vector of 1 and 0 . In those cells a diffusing molecule 
undergoes a degradation equal to its diffusion rate $D$. This term approximates a continued flux into the non-modelled larger tissue below the meristem. A diffusing molecule is also degraded with a rate $g$.

For a cell vector of concentrations of a diffusing molecule $X$, we have:

$$
\frac{d X}{d t}=p P-g X+D \Delta X-D B X
$$

where $\Delta$ is the Laplace operator; transport in the model is assumed to be passive. For a cell $i$ with $n_{i}$ neighbours $\{j\}$, the diffusion of $X$ follows:

$$
\frac{d X_{i}}{d t}=D \sum_{j}^{n_{i}}\left(X_{j}-X_{i}\right)
$$

The equilibrium state of the considered molecule is found by solving the equation:

$$
p P-g X+D \Delta X-D B X=0
$$

In the present study, this is achieved using the linalg.solve function of the python scientific package scipy.

\section{Model}

The complete model describes the interactions between the components of the system represented in Supplementary Fig. 8. ZmWUS1 expression is driven by cytokinin binding the ZmAHK4 receptors. FEA3 receptors upon binding $\mathrm{ZmFCP1}$, and FEA2 receptors upon binding either ZmCLV3 or ZmFCP1 (as supported by peptide assays in Fig 3a and Supplementary Fig. 6b), repress ZmWUS1 expression. FEA2 is constitutively expressed in the model ${ }^{10}$. ZmCLV3 expression is activated by ZmWUS1 and a hypothetical L1 originating signal. A schematic description of the model is shown in

\section{Supplementary Fig. 8.}


A set of seven differential equations for each cell controls the behaviour of the genes $\operatorname{ZmWUS1}(W)$ and $Z m C L V 3(C)$, WUS protein $(w)$, the ZmCLV3 (c) and ZmFCP1 $(f)$ peptides, cytokinin $(\gamma)$ and the $\mathrm{L} 1$ originating $\mathrm{ZmCLV3}$ activator $(l)$, variables in brackets indicate molecular concentrations:

$$
\begin{gathered}
\frac{d[W]}{d t}=V_{W} \times \frac{([\gamma][A])^{n_{W}}}{([\gamma][A])^{n_{W}}+k_{A}^{n_{W}}} \times \frac{k_{F_{3}}^{n_{W}}}{\left(\left[F_{3}\right][f]\right)^{n_{W}}+k_{F_{3}}^{n_{W}}} \times \frac{k_{F_{2}}^{n_{W}}}{([c]+[f])^{n_{W}+k_{F_{2}}^{n_{W}}}-g_{W}[W]} \\
\frac{d[w]}{d t}=p_{w}[W]-g_{w}[w]+D_{w} \Delta[w]-D_{w} B[w] \\
\frac{d[C]}{d t}=V_{C} \times \frac{[l]^{n_{C}}}{[l]^{n_{C}}+k_{l}^{n_{C}}} \times \frac{[w]^{n_{C}}}{[w]^{n_{C}}+k_{w}^{n_{C}}}-g_{C}[C] \\
\frac{d[c]}{d t}=p_{c}[C]-g_{c}[c]+D_{c} \Delta[c]-D_{c} B[c] \\
\frac{d[f]}{d t}=p_{f}[F]-g_{f}[f]+D_{f} \Delta[f]-D_{f} B[f] \\
\frac{d[l]}{d t}=p_{l} L 1-g_{l}[l]+D_{l} \Delta[l]-D_{l} B[l] \\
\frac{d[\gamma]}{d t}=p_{\gamma} L 1-g_{\gamma}[\gamma]+D_{\gamma} \Delta[\gamma]-D_{\gamma} B[\gamma]
\end{gathered}
$$

$A$ is $\mathrm{ZmAHK} 4, F_{3}$ is FEA3, $F_{2}$ is FEA2, and $F$ is the $Z m F C P 1$, all statically defined (Supplementary

Fig. 7). The complete system comprises 5642 equations.

\section{Parameter values}

Since parameter values are unknown they are inferred via an optimization strategy, adapted from ${ }^{28}$. In order to maintain the computations linear, we propose a workaround the CLV3-WUS feedback loop by optimizing the system in several steps, where subparts of the network are optimized and then merged. All optimisations are carried out using the CMA-ES algorithm ${ }^{50}$. The strategy can be summarized as: (i) optimize the ZmWUS1 domain using the static ZmCLV3 domain $(C t)$ as defined in the template (Supplementary Fig. 7), (ii) optimize the $Z m C L V 3$ domain keeping the optimized ZmWUS1 domain fixed, (iii) optimize the ZmCLV3 gradient such that the optimized ZmCLV3 domain produces a gradient as similar as possible to the one resulting from the first step. This is followed by a confirmation step (iv), where the equilibrium of the complete optimized network is computed.

In the following, $\operatorname{diff}(P)$ will refer to computing the equilibrium concentration of a diffusing molecule across the tissue, given a production domain $P$. Similarly, $e q\left(\left[A_{1} \ldots A_{N a}\right],\left[I_{1} \ldots I_{N b}\right]\right)$ will refer to 
computing the equilibrium of a gene expression regulated by $\mathrm{Na}$ activators and $\mathrm{Nb}$ inhibitors (equations above). Finally, the function $\operatorname{mes}(X, Y,[\varphi, \omega])$ measures the difference between two vectors $\mathrm{X}$ and $\mathrm{Y}$ following: $\sum_{i}\left(X_{i}-Y_{i}\right)^{2}$, and is the main part of the cost functions used in the different steps of the optimisation strategy. If cell $i$ of either vector meets the condition $\varphi$, the value of $\left(X_{i}-Y_{i}\right)^{2}$ is weighted by parameter $\omega$ in the final sum. The condition is used to balance the relative value of cells expressing a gene against cells not expressing it when a small proportion of the total amount of cells express the gene.

(i) ZmWUS1 expression domain. The optimisation minimises the difference between $Z m W U S 1$ as defined in the template $\left(W_{t}\right)$ and $\operatorname{ZmWUS1}(W)$ at equilibrium, a second component of the cost function ensures that the ZmWUS1 domain $\left(W_{F 2}\right)$ is enlarged in fea2 compared to wild type, and a third component that ZmWUS1 $\left(W_{F 3}\right)$ resembles the target ZmWUS1 domain in fea3 ( $\left.W_{t F 3}\right)$. The cost function, $E_{W}$, is defined by:

$$
E_{W}=\operatorname{mes}\left(W, W_{t},\left[W_{t}=1,5\right]\right)+\operatorname{mes}\left(W_{F 3}, W_{t F 3}\right)+\left(0 \text { if } \frac{\sum W_{F 2}}{\sum W}<0.75 \text { else } \frac{\sum W_{F 2}}{\sum W} \times 40\right)
$$

where the summations are over all cells. Computation of ZmWUS1 equilibrium follows:

$$
\begin{gathered}
f=\operatorname{diff}(F) \\
c_{h}=\operatorname{diff}\left(C_{t}\right) \\
W=e q\left([\gamma A],\left[\left(c_{h}+f\right),\left(F_{3} \times f\right)\right]\right)
\end{gathered}
$$

ZmWUS1 equilibrium in fea2:

$$
W_{F 2}=e q\left([\gamma A],\left[\left(F_{3} \times f\right)\right]\right)
$$

ZmWUS1 equilibrium in fea3:

$$
W_{F 3}=e q\left([\gamma A],\left[\left(c_{h}+f\right)\right]\right)
$$

The parameters $k_{A}, k_{F 3}, k_{F 2}, g_{f}, D_{f}, g_{c}$ and $D_{c}$ are optimised. $g_{W}, p_{c}$ and $p_{f}$ are set to $1, n_{W}$ is set to 2 and $V_{W}$ to $1.5 \cdot p_{\gamma}, g_{\gamma}$ and $D_{\gamma}$ are set to 1,1 and 50 , respectively. 
(ii) $\mathrm{ZmCLV3}$ expression domain. The optimisation aims at minimising the difference between $Z m C L V 3$ at equilibrium and $\mathrm{C}_{\mathrm{t}}$. A second component of the cost function ensures the expression domain of $Z m C L V 3$ increases with an increase of WUS.

The equilibrium of $\mathrm{ZmCLV3}$ is computed as follows:

$$
\begin{gathered}
w=\operatorname{diff}(W) \\
l=\operatorname{diff}(L 1) \\
C=e q([l, w],[]) \\
C_{2}=e q([l, w \times 2],[])
\end{gathered}
$$

and the cost function is:

$$
E_{C}=\operatorname{mes}\left(C, C_{t},\left[C_{t}=1,2\right]\right)+20 \frac{C^{6}}{C_{2}^{6}}
$$

The parameters $k_{l}, k_{w}, g_{w}$ and $D_{w}$ are optimised. $V_{C}$ is set to $1.5, n_{C}$ to 10 and $g_{C}$ to $1 . p_{w}, p_{l}, g_{l}$ and $D_{l}$ are set to 1 .

(iii) ZmCLV3 peptide gradient. The first part of the optimisation used a hypothetical ZmCLV3 signal $\left(c_{\mathrm{h}}\right)$ produced by the manually defined $Z m C L V 3$ expression domain. As an equilibrium $Z m C L V 3$ domain has been computed, one can compute the corresponding equilibrium for the ZmCLV3 peptide. Optimising ZmCLV3 aims at minimising its difference with the previously computed $c_{\mathrm{h}}$.

Equilibrium for ZmCLV3 follows:

$$
c=\operatorname{diff}(C)
$$

and the cost function is:

$$
E_{c}=\operatorname{mes}\left(c, c_{h}\right)
$$

Parameters $g_{c}$ and $D_{c}$ are re-optimised in this step. 
(iv) $Z m C L V 3 / Z m W U S$ feedback loop - Equilibrium of the complete model. As the previous steps of the optimisation independently found equilibria for components of the model, one has to ensure an adequate stable state exists also for the complete model. The feedback between ZmWUS1 and ZmCLV3 tends to induce dampened oscillations of the two genes. We propose an algorithm making use of that aspect and designed to quickly reach the equilibrium of the system using the parameter values obtained in the previous steps:

$$
\begin{aligned}
& E_{d}=\sum^{i}\left(\frac{d w_{i}{ }^{2}}{d t}+\frac{d c_{i}{ }^{2}}{d t}\right) \\
& \text { while } E_{d}>T_{0}: \\
& \qquad \begin{aligned}
\sum^{i} \frac{d w_{i}}{d t} & <\sum^{i} \frac{d c_{i}{ }^{2}}{d t}: \\
W & =e q\left([A],\left[(c+f),\left(F_{3} \times f\right)\right]\right) \\
w & =\frac{d i f f(W)+w}{2} \\
C & =e q([l, w],[]) \\
c & =\frac{d i f f(C)+c}{2}
\end{aligned}
\end{aligned}
$$

else:

$$
\begin{gathered}
C=e q([l, w],[]) \\
c=\frac{d i f f(C)+c}{2} \\
W=e q\left([A],\left[(c+f),\left(F_{3} \times f\right)\right]\right) \\
W=\frac{d i f f(W)+w}{2} \\
E_{d}=\Sigma^{i}\left(\frac{d w_{i}^{2}}{d t}+\frac{d c_{i}^{2}}{d t}\right)
\end{gathered}
$$

If $E_{d}$ falls below a defined threshold $T_{0}$, the model is considered to have reached equilibrium. Additional numerical simulations confirm the equilibria are correctly found by this algorithm.

\section{Behaviour of the model}

Multiple optimisations resulted in 203 parameter sets successfully describing the wild type expression of ZmWUS1 and ZmCLV3. In order to assess the ability of the model to describe perturbations of the system, a set of mutants were computed for all the optimised parameter sets:

- Zmclv3:

$$
P_{c} \rightarrow 0
$$


- fea2:

$$
\frac{d[W]}{d t} \rightarrow V_{W} \times \frac{([\gamma][A])^{n_{W}}}{([\gamma][A])^{n_{W}}+k_{A}^{n_{W}}} \times \frac{k_{F_{3}}^{n_{W}}}{\left(\left[F_{3}\right][f]\right)^{n_{W}}+k_{F_{3}}^{n_{W}}}-g_{W}[W]
$$

- fea3:

$$
F_{3} \rightarrow \mathrm{F}_{3} \times 0
$$

- fea2/fea3: implements both fea2 and fea3 mutations

- $f_{c p 1:}$

$$
p_{f} \rightarrow 0
$$

- $\quad f c p 1$ over-expression:

$$
p_{f} \rightarrow p_{f} \times 1.5
$$

Both the wild type expression domains of ZmWUS1 and ZmCLV3 along with their expression in mutants are exemplified in Supplementary Fig. 9. The variation of the expression of the two genes, between wild type and mutant phenotypes and for all parameter sets, is presented in Supplementary Fig. 10.

fea 2, fea 3 and fea3; fea 2 all lead to a substantial increase of both ZmWUS1 and ZmCLV3, the model thus predicts a fasciated phenotype for the three mutants, which proves to be consistent with the experimental data presented in Fig. 1. Moreover, as shown, the severity of the phenotypes increases from fea 2 to fea 3 to fea $2 / f e a 3$. Both the fea 3 and fea 3 ; fea 2 mutants result in a large downward expansion of the ZmWUS1 domain, similar to the $p Z m W U S 1-N L S . R F P$ expression domain observed in fea 3 (Fig. 2).

$f c p 1$ causes a large expansion of ZmWUS1 and ZmCLV3 and a predicted fasciated phenotype. The ZmWUS1 domain exhibits a downward expansion typical of the fea 3 mutants. 
Finally, over-expressing the $f c p 1$ peptide in its own expression domain (using a primordium specific YABBY promoter) causes a decrease of ZmWUS1 expression, leading to a reduction of ZmCLV3 expression. The phenotype suggests a smaller stem cell pool and thus a reduction of the meristem size or meristem arrest.

A notable aspect shared by all optimised parameter sets is the relatively weak feedback from $Z m C L V 3$ to ZmWUS1 (cf. Supplementary Fig. $10-z m c l v 3$, showing that ZmWUS1 expression is mostly unaffected by the zmclv 3 phenotype). In order to achieve a correct fea 2 phenotype, the optimisation has to increase the expression of ZmWUS1 when FEA2 receptors are lost. As the model allows both zmclv3 and fcp1 peptides to repress ZmWUS1 expression, any balance of effects mediated by those two peptides could theoretically mediate the phenotype. However, after pooling all optimisation constraints together, resulting parameter sets mediate fea 2 phenotype mostly via fcp 1 . This is caused by the constraint on fea 3 phenotype, where ZmWUS1 has to expand downward. Upon loss of FEA3 receptors, the expansion of ZmWUS1 causes an expansion of the stem cell domain and an increase of the production of zmclv3. Would zmclv3 be a strong repressor of ZmWUS1, ZmWUS1 would be repressed away from the tip of the meristem; this would contradict the experimental data presented on Fig. 2d. The solution to conciliate $f e a 2$ and $f e a 3$ phenotypes is to mediate $f e a 2$ phenotype by fcp 1 while tuning down the effect of zmclv3. This suggests that, in maize, the repression of ZmWUS1 by the stem cell domain (mediated by zmclv3) may be weak or nonexistent, contrary to $A$. thaliana.

Together with the parameter values fixed in the optimization as described above, optimised parameter values used to exemplify the behaviour of the model in Fig. 3 and Supplementary Fig. 7-10 are:

$$
\begin{aligned}
& k_{A}=0.0799349478204 \\
& k_{F_{3}}=0.258483108091 \\
& k_{F_{2}}=0.631322009895 \\
& g_{f}=0.0989706220105 \\
& D_{f}=0.315196037043 \\
& k_{l}=0.35868731223 \\
& k_{w}=1.28418451672
\end{aligned}
$$




$$
\begin{aligned}
& g_{w}=10^{-5} \\
& D_{w}=3.05859740889 \\
& g_{c}=1.41162777475 \\
& D_{c}=0.795496002536
\end{aligned}
$$

\section{"Rice-like" model.}

The model described above is largely inspired by data obtained in A. thaliana. In particular, the $L O G$ genes, whose expression patterns are unknown in maize, are expressed in the epidermis; in rice the expression resembles the stem cell domain ${ }^{51}$. In order to assess the importance of this choice we modified the model so the production of active cytokinin would not be limited to the epidermis, but to the tip of the meristem, as suggested from the data obtained in rice.

To assess this second model we followed the same procedure as described for the "Arabidopsis-like" model, both for the optimisation of the model and for the mutant analysis. The single difference between the two procedures is the source of cytokinin: L1 in the "Arabidopsis-like" model, ZmCLV3 target in the "rice-like" model (Supplementary Fig. 7).

The "Rice-like" model correctly generates the wild type and mutant expression domains (Supplementary Fig. 11). This holds true for the 295 sets of parameters resulting from the optimisation procedure (Supplementary Fig. 12). Strikingly, the "rice-model" behaves extremely similarly to the "Arabidopsis model", suggesting that the pattern of expression of the LOGs in maize could be similar to either organisms without affecting the conclusions of the present study.

The model parameters exemplified in Supplementary Fig. 11, 12 are:

$$
\begin{aligned}
& k_{a}=0.0110285146717 \\
& k_{F_{3}}=0.308187217119 \\
& k_{F_{2}}=0.9037413184 \\
& g_{f}=0.078395885527
\end{aligned}
$$




$$
\begin{aligned}
D_{f} & =0.256708058963 \\
k_{l} & =0.358739560589 \\
k_{w} & =0.723764685978 \\
g_{w} & =1 e-05 \\
D_{w} & =5.3995729066 \\
g_{c} & =0.697132853884 \\
D_{c} & =0.519479366051
\end{aligned}
$$

\section{References}

57. Winter, D. et al. An "Electronic Fluorescent Pictograph" browser for exploring and analyzing largescale biological data sets. PLoS One 2, e718 (2007).

58. Yadav, R.K., Girke, T., Pasala, S., Xie, M. \& Reddy, G.V. Gene expression map of the Arabidopsis shoot apical meristem stem cell niche. Proc Natl Acad Sci U S A 106, 4941-6 (2009). 